\title{
Tetrahydrofolate Alleviates the Inhibitory Effect of Oxidative Stress on Neural Stem Cell Proliferation through PTEN/Akt/ mTOR Pathway
}

\author{
Xuyang Zhang $\mathbb{D}^{1}{ }^{1}$ Zhi Liu, ${ }^{1}$ Wenqin Yang, ${ }^{1}$ Fengchun Zhao, ${ }^{1}$ Chao Zhang, ${ }^{1}$ Hui Feng, \\ Tengyuan Zhou, ${ }^{1}$ Jun Zhong $\mathbb{D}^{1},{ }^{1}$ Yongjie Zou, ${ }^{2}$ Hua Feng, ${ }^{1}$ Hongfei Ge $\mathbb{D}^{1},{ }^{1}$ and Rong Hu ${ }^{1}$ \\ ${ }^{1}$ Department of Neurosurgery and Key Laboratory of Neurotrauma, Southwest Hospital, Third Military Medical University (Army \\ Medical University), 400038 Chongqing, China \\ ${ }^{2}$ Department of Neurosurgery, The 908th Hospital of Chinese People's Liberation Army Joint Logistic Support Force, \\ 330002 Nanchang, Jiangxi, China
}

Correspondence should be addressed to Hongfei Ge; hongfei0723@163.com and Rong Hu; huchrong@tmmu.edu.cn Received 10 November 2021; Accepted 10 February 2022; Published 27 February 2022

Academic Editor: Francisco J. Romero

Copyright (C) 2022 Xuyang Zhang et al. This is an open access article distributed under the Creative Commons Attribution License, which permits unrestricted use, distribution, and reproduction in any medium, provided the original work is properly cited.

\begin{abstract}
Neural stem cell (NSC) proliferation is the initial step for NSC participating in neurorehabilitation after central nervous system (CNS) injury. During this process, oxidative stress is always involved in restricting the regenerative ability of NSC. Tetrahydrofolate (THF) is susceptible to oxidative stress and exhibits a high antioxidant activity. While its effect on NSC proliferation under oxidative stress condition remains obscure. Here, NSC were isolated from embryonic mice and identified using immunofluorescent staining. Meanwhile, the results showed that THF $(5 \mu \mathrm{M}$ and $10 \mu \mathrm{M})$ attenuated oxidative stress induced by $50 \mu \mathrm{M}$ hydrogen peroxide $\left(\mathrm{H}_{2} \mathrm{O}_{2}\right)$ in NSC using mitochondrial hydroxyl radical detection and Western blotting assays. Afterward, administration of THF markedly alleviated the inhibitory effect of oxidative stress on NSC proliferation, which was evidenced by Cell Counting Kit-8 (CCK8), neurosphere formation, and immunofluorescence of Ki67 assays. Thereafter, the results revealed that PTEN/Akt/mTOR signaling pathway played a pivotal role in counteracting oxidative stress to rescue the inhibitory effect of oxidative stress on NSC proliferation using Western blotting assays and gene knockdown techniques. Collectively, these results demonstrate that THF mitigates the inhibitory effect of oxidative stress on NSC proliferation via PTEN/Akt/mTOR signaling pathway, which provides evidence for administrating THF to potentiate the neuro-reparative capacity of NSC in the treatment of CNS diseases with the presence of oxidative stress.
\end{abstract}

\section{Introduction}

Neural stem cell (NSC), residing in the dentate gyrus (DG) of hippocampus and subventricular zone (SVZ) of the lateral ventricle, are the main regenerative neural cell subtype in the central nervous system (CNS) [1-4]. Under physiological condition, NSC dynamically keep in a delicate balance among self-renewal, differentiation, and quiescence [5]. While under pathological situation, NSC hold the ability of proliferating in situ, migrating toward the injured regions, differentiating into three main neural subpopulations (neurons, astrocytes, and oligodendrocytes), and integrating into the damaged neurovascular network to accelerate functional recovery after CNS injury [6-9]. NSC proliferation is the initial step for NSC taking part in neurorehabilitation after CNS injury. Several factors have been identified to be associated with NSC proliferation.

The level of reactive oxygen species (ROS) is one of the factors mediating NSC proliferation. At steady state, low basal dynamic ROS levels maintain NSC in a sophisticated balance among self-renewal, differentiation, and quiescence [5]. Previous studies have indicated that a slightly higher ROS level boosts NSC proliferation isolated from SVZ [5, 10-12], whereas excessive level of ROS, characterized as ROS surplus, always results in oxidative stress [13]. When oxidative stress persists, NSC are prone to suffer from 
maintaining in quiescence, that is, NSC lose proliferation potential or lack of self-renewal capacity $[13,14]$. Considering that oxidative stress is often introduced in subjects with the ictus and progression of CNS injury, such as traumatic brain injury (TBI) $[15,16]$, cerebral stroke [17-19], and neurodegenerative diseases [20-22], exploring interventions to alleviate oxidative stress might be an effective therapeutic strategy to strengthen the rehabilitative ability of NSC after CNS injury.

Tetrahydrofolate (THF), the active form of folate (vitamin B9), is an essential cofactor of one-carbon (1C) cycle and involved in the biosynthesis of thymidylate, purines, and glycine [23, 24]. Previous researches have delineated that THF is susceptible to oxidative stress and exhibits a high antioxidant activity when excessive level of ROS cumulation is available $[25,26]$, implying that THF might be a candidate for ameliorating surplus ROS to enhance rehabilitative capacity of NSC. Meanwhile, previous study has demonstrated that the THF transporter of SLC25A32 is highly expressed in varying tumors, and obviously facilitated tumor cell growth to reduce patients' survival, whereas downregulation of SLC25A32 inhibits tumor cell propagation [27]. Moreover, THF administration is an effective intervention in promoting proliferation of dihydrofolate reductase(DHFR-) deficient Chinese hamster ovary (CHO) DG44 cells [24], while the effect of THF on NSC proliferation and the underlying mechanism remains largely unknown.

Phosphatase and tensin homologue (PTEN) usually serves as a negative modulator in maintaining NSC proliferation [28]. Recently, investigation shows that PTEN/protein kinase $\mathrm{B}$ (Akt) signaling pathway exerts neuroprotective effect on spiral ganglion neuron after oxidative stress exposure in a manner of negatively regulating Akt [29]. Additionally, previous studies have demonstrated that Akt/mammalian target of rapamycin (mTOR) signaling pathway is an essential regulator for NSC proliferation [30-33] and participates in manipulating redox homeostasis in macrophage and glioma cells [34-36]. Thereafter, a hypothesis that THF administration might mitigate the inhibitory effect of oxidative stress on NSC proliferation due to PTEN/Akt/mTOR pathway is issued in the present study.

To test this hypothesis, NSC were isolated from embryonic mice and identified using phase-contrast observation and immunofluorescence. Next, the hydrogen peroxide $\left(\mathrm{H}_{2} \mathrm{O}_{2}\right)$ was used to introduce oxidative stress, and the level of mitochondrial ROS (MitoROS) was determined using mitochondrial hydroxyl radical detection assays. Afterward, the effect of THF on NSC proliferation under oxidative stress condition was determined by Cell Counting Kit- 8 (CCK8), neurosphere formation, and immunofluorescence of Ki67 assays. Subsequently, the underlying mechanism was explored using Western blotting assays and gene knockdown techniques. The aim of the present study is to provide a rationale for administrating THF to potentiate the restorative ability of NSC in the treatment of CNS diseases with the presence of oxidative stress.

\section{Materials and Methods}

2.1. Animals. Embryonic C57BL/6 mice were purchased from the Third Military Medical University (Army Medical
University). All animal experiments were performed in accordance with China's animal welfare legislation for protection of animals used for scientific research and were approved by the local authorities of the Third Military Medical University (Army Medical University) for the laboratory use of animals (approval no. SCXK-20170002).

2.2. NSC Culture and Treatment. Primary NSC were isolated and cultured as previously described [9, 37]. Briefly, the cerebral cortex was dissected and washed twice with DMEM (Gibco, Grand Island, NY). After peeling off the meninges, tissues were incubated in $0.25 \%$ trypsin-EDTA (Gibco, Grand Island, NY) at $37^{\circ} \mathrm{C}$ for 30 minutes, then washed twice in DMEM after being incubating in $10 \%$ fetal bovine serum (FBS, Gibco, Grand Island, NY) for 2 times. Finally, tissues were suspended in stem cell culture medium as follows: DMEM/F12 medium supplemented with 2\% B27 (Gibco, Grand Island, NY), $20 \mathrm{ng} / \mathrm{ml} \mathrm{EGF}$ (Peprotech, Rocky Hill, NJ) and 20 ng/ml FGF-2 (Peprotech, Rocky Hill, NJ). Afterward, cell suspension was passed through a $70 \mu \mathrm{m}$ Nylon cell strainer (BD Falcon, San Jose, CA). Subsequently, NSC suspension was incubated at $37^{\circ} \mathrm{C}$ under $5 \% \mathrm{CO}_{2}$ humidified condition. The procedures for NSC passage were performed according to standard methods as described in our previous researches $[9,37]$. In order to make NSC grow adherently, $10 \mu \mathrm{g} / \mathrm{ml}$ poly-L-ornithine (Sigma-Aldrich, St. Louis, MO) was used to precoat cell culture plates. NSC were allowed to grow at a density of $1 \times 10^{5}$ for 24 hours at $37^{\circ} \mathrm{C}$ prior to implementing the experiments. NSC were pretreated with THF for 30 minutes and then stimulated with $50 \mu \mathrm{M} \mathrm{H}_{2} \mathrm{O}_{2}$. THF (Sigma-Aldrich, St. Louis, MO) and $\mathrm{H}_{2} \mathrm{O}_{2}$ (Lircon, Shandong, China) were dissolved in PBS, and diluted to specific concentration by NSC culture medium. Meanwhile, the equal volume of PBS was added as control or scramble group.

2.3. NSC Differentiation. NSC were seeded on confocal culture dishes precoated with $10 \mu \mathrm{g} / \mathrm{ml}$ poly-L-ornithine (PLO). Then, cells were incubated in DMEM/F12 containing $1 \%$ glutamate (Gibco, Grand Island, NY) and 2\% B27 (Gibco, Grand Island, NY) for 7 days.

2.4. Cell Viability Assays. Cell Counting Kit-8 (CCK8) assays were performed to assess the viability of NSC. Approximately $2 \times 10^{4}$ single NSC in $200 \mu \mathrm{l}$ culture medium were seeded in 96-well plates for 24 hours, then treated with different concentrations of THF. Subsequently, $50 \mu \mathrm{M} \mathrm{H} \mathrm{H}_{2} \mathrm{O}_{2}$ was added after 30 minutes. Thereafter, $20 \mu \mathrm{l} \mathrm{CCK-8} \mathrm{work-}$ ing solution (Boster, Wuhan, China) was added into each well and incubated at $37^{\circ} \mathrm{C}$ for 2 hours. The absorbance value was determined with a microplate reader at $450 \mathrm{~nm}$.

2.5. Growth Curve Analysis. NSC were seeded in 12-well plates at a density of $1 \times 10^{5}$ single cell per well. Then, NSC in each group were treated with $\mathrm{H}_{2} \mathrm{O}_{2}$ or THF for 0 , 24, 48, and 72 hours. Thereafter, cells were counted with a haemocytometer after being suspended in culture medium. 
$6 \mathrm{~h}$
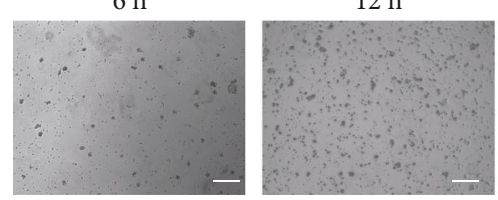

$24 \mathrm{~h}$

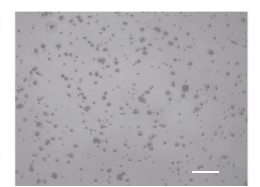

(a)

DAPI/Nestin /Sox2
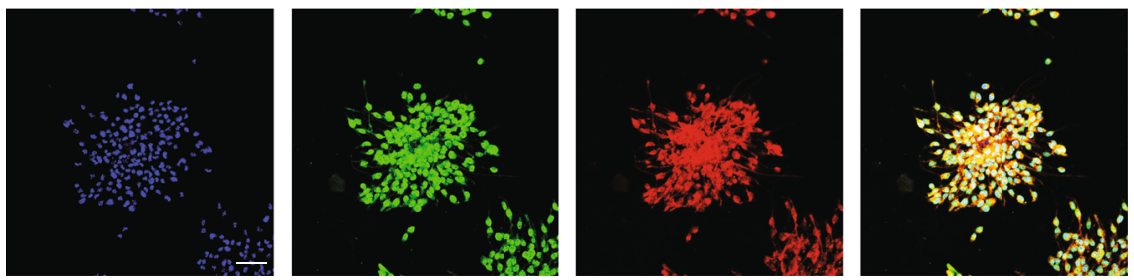

$48 \mathrm{~h}$
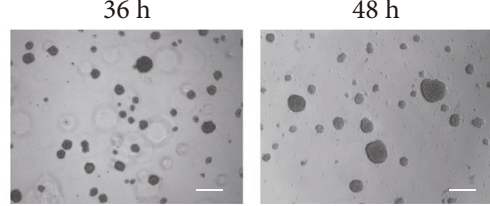

(b)

DAPI / GFAP
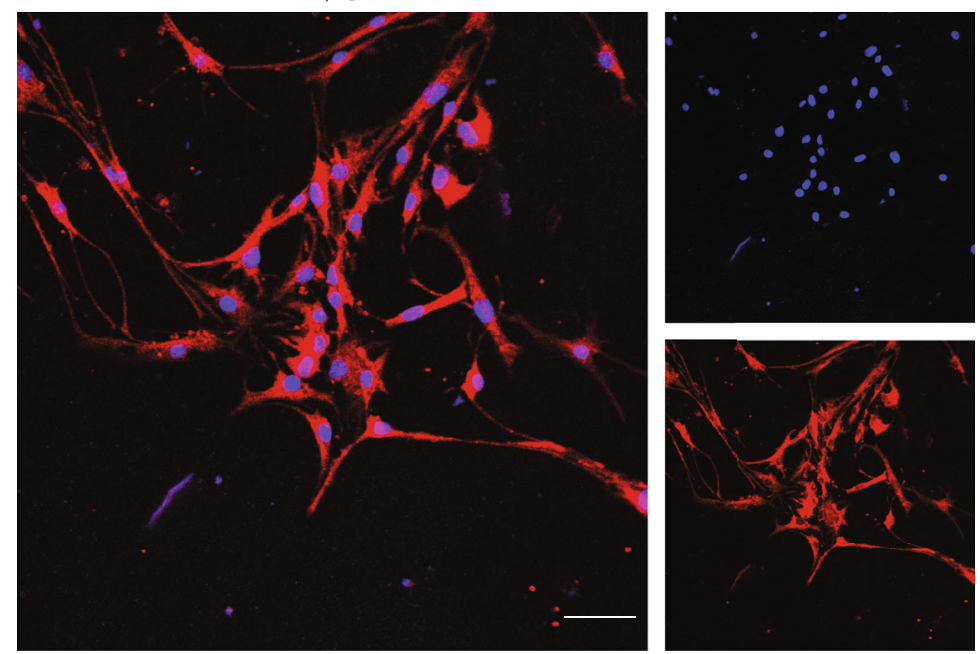

(c)

DAPI / MAP2
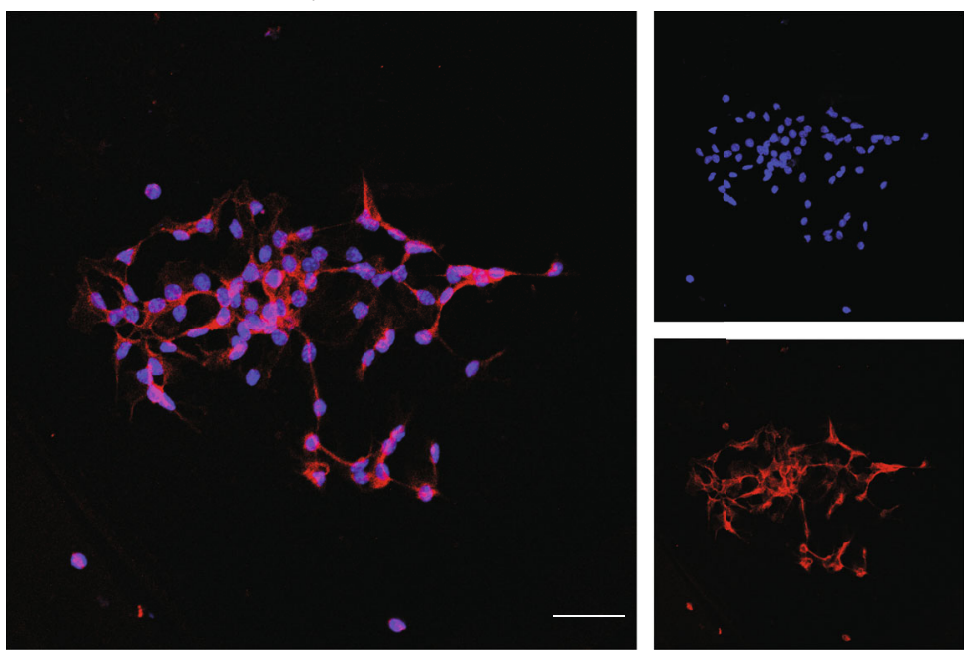

(d)

Figure 1: Continued. 


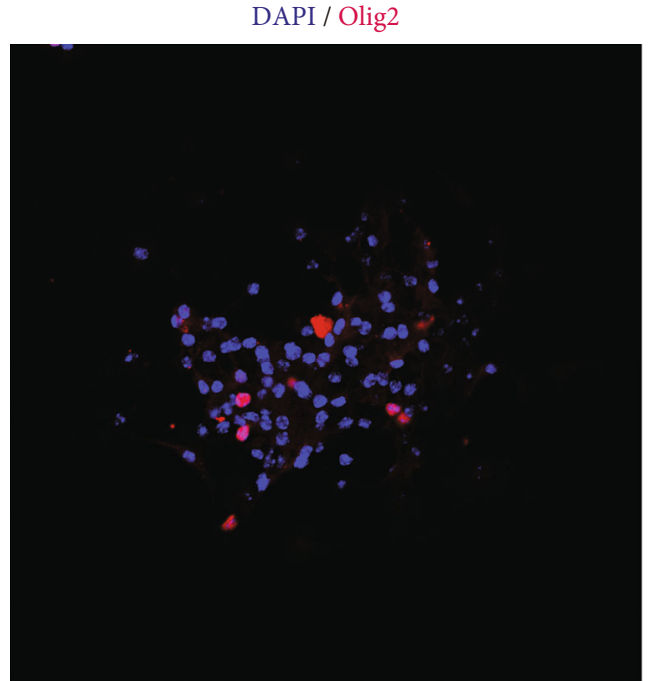

(e)
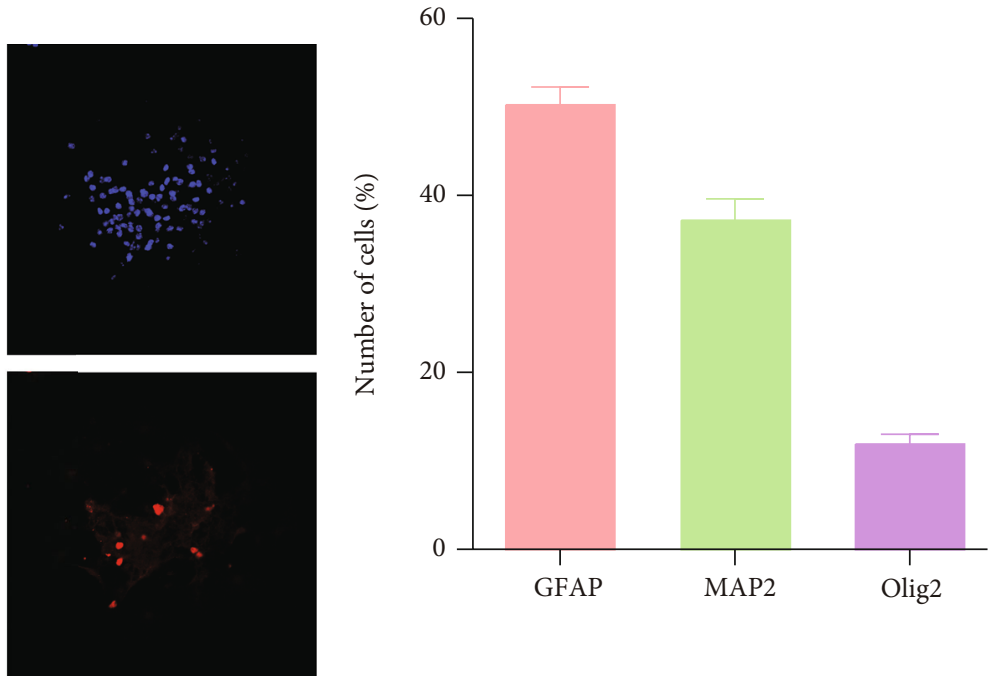

(f)

Figure 1: NSC culture and characteristics. (a) Morphological changes of cultured NSC under phase-contrast microscope from 6 to 48 hours in floating condition. Scale bar: $50 \mu \mathrm{m}$. (b) Immunostaining images represented that the majority of cultured cells were positive for Nestin and/or Sox2. Scale bar: $20 \mu \mathrm{m}$. (c) Immunostaining images showed that cultured cells could differentiate into GFAP ${ }^{+}$cells after 7 days in differentiation medium. Scale bars: $10 \mu \mathrm{m}$. (d) Immunostaining pictures indicated that cultured cells held the potential of differentiation into $\mathrm{MAP}^{+}$cells after 7 days in differentiation medium. Scale bars: $10 \mu \mathrm{m}$. (e) Immunostaining images presented cultured cells possessed the capacity of transformation into Olig2 ${ }^{+}$cells after 7 days in differentiation medium. Scale bars: $10 \mu \mathrm{m}$. (f) Histogram summarizing the percentage of cultured cell differentiation into $\mathrm{GFAP}^{+}, \mathrm{MAP}^{+}$, and $\mathrm{Olig}^{+}$cells, respectively.

2.6. Mitochondrial Hydroxyl Radical Detection. The test was performed according to the manufacturers' instructions of Cell Meter ${ }^{\mathrm{TM}}$ Mitochondrial Hydroxyl Radical Detection Kit (AAT Bioquest, USA). Briefly, NSC were seeded onto $10 \mu \mathrm{g} / \mathrm{ml}$ PLO-coated confocal culture dishes and incubated for 24 hours. Then, they were incubated in $\mathrm{H}_{2} \mathrm{O}_{2}$ or THF for another 24 hours. Afterward, the culture medium was removed, and $1 \mathrm{ml}$ MitoROS ${ }^{\mathrm{TM}} \mathrm{OH} 580$ working solution was added to each confocal dish at $37^{\circ} \mathrm{C}$ for 60 minutes. Thereafter, cells were washed 2-3 times with Hank's Balanced Salt Solution (HBSS), and Hoechst dye solution (Beyotime, China) was diluted to each well for 15 minutes. The fluorescence density was determined using a Confocal Laser Microscopy (Carl Zeiss, LSM880, Weimar, Germany) at $\mathrm{Ex} / \mathrm{Em}=540 / 590 \mathrm{~nm}$ with bottom read mode and examined by Zen 2011 software (Carl Zeiss, Weimar, Germany).

2.7. Neurosphere Formation Assay. Approximate amount of 1000 single NSC were cultured in uncoated 12-well plate for 24 hours. NSC in each group were suffered from different treatments. Then, the number and average diameter were observed using a phase-contrast microscopy (IX71, Olympus, Tokyo) at 24 hours and 48 hours.

2.8. PTEN Knockdown. The procedures for PTEN knockdown were performed according to the manufacturer's instruction. PTEN shRNA lentivirus were purchased from Beijing Syngentech Co., Ltd. (Beijing, China). NSC were infected with PTEN-1 shRNA lentivirus at 70 multiplicity of infection (MOI) or PTEN-2 shRNA lentivirus at 100 MOI. Then, the PTEN knockdown efficiency was assessed using Western blotting assays at 4 days. The following target sequences were used in the present experiments: PTEN-1: CGACTTAGACTTGACCTATAT; PTEN-2: TTGTGG CAACAGATAAGTTTG.

2.9. Immunofluorescence. NSC were cultured in separated confocal culture dishes with different treatments. Afterward, NSC adhered to confocal culture dishes were fixed with $4 \%$ paraformaldehyde (PFA) for 30 minutes at room temperature and followed by $0.5 \%$ Triton X-100 in PBS for 30 minutes, then blocked with 5\% BSA for 2 hours after washing with PBS for three times. After that, the cells were incubated with mouse anti-Nestin (1:200, Abcam, Cambridge, UK), rabbit anti-Sox2 (1:100, CST, USA), rabbit anti-Ki67 ( $1: 400$, CST, USA), rabbit anti-GFAP $(1: 800$, Abcam, Cambridge, UK), rabbit anti-MAP2 (1:400, CST, USA), or rabbit anti-Olig2 (1:100, Proteintech Group, Inc., Chicago, IL, USA) antibodies overnight at $4^{\circ} \mathrm{C}$. After washing with PBS for three times, the corresponding fluorescence secondary antibodies were incubated for 2 hours at room temperature, then cell nuclei were counterstained with $4^{\prime}, 6$-diamidino-2phenylindole (DAPI) for 20 minutes at room temperature. Finally, samples were visualized with a confocal microscope (Carl Zeiss, LSM780, Weimar, Germany) and examined by a Zen 2011 software (Carl Zeiss, Weimar, Germany).

2.10. Western Blotting. Samples in different groups were lysed by radio immunoprecipitation assay lysis buffer (RIPA; Beyotime, China) containing protease and phosphatase inhibitor cocktail (Roche, Indianapolis, IN, USA). Proteins of the same quantity ( $30 \mu \mathrm{g} /$ lane) in each group were separated by $10 \%$ or $15 \%$ SDS-PAGE and transferred to polyvinylidene difluoride (PVDF) membranes (Millipore, 

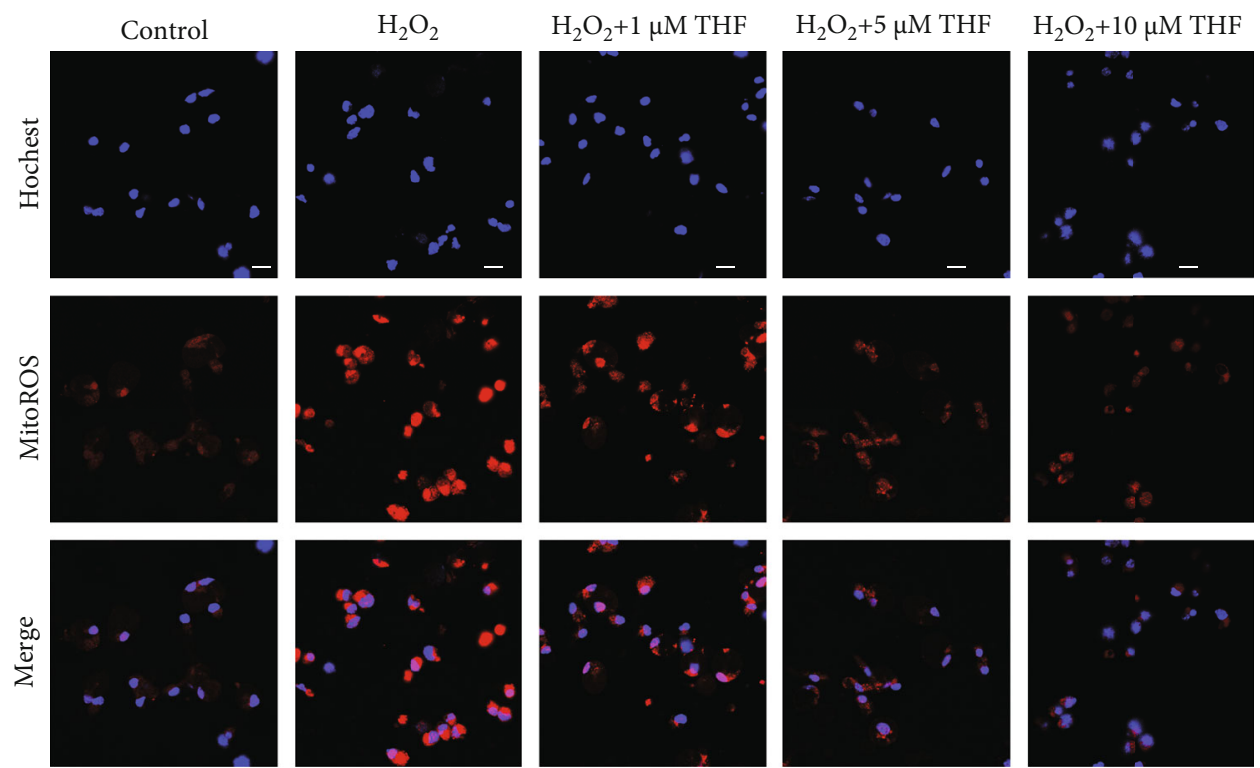

(a)

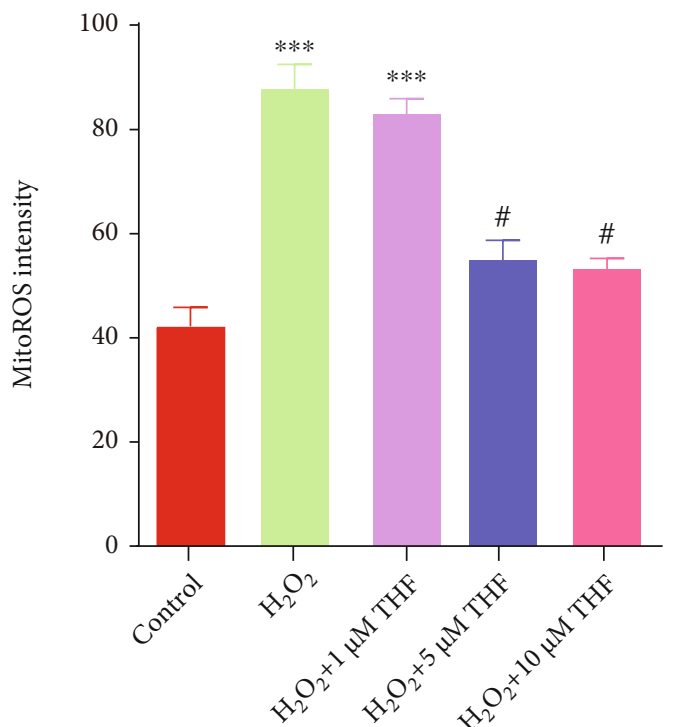

(b)

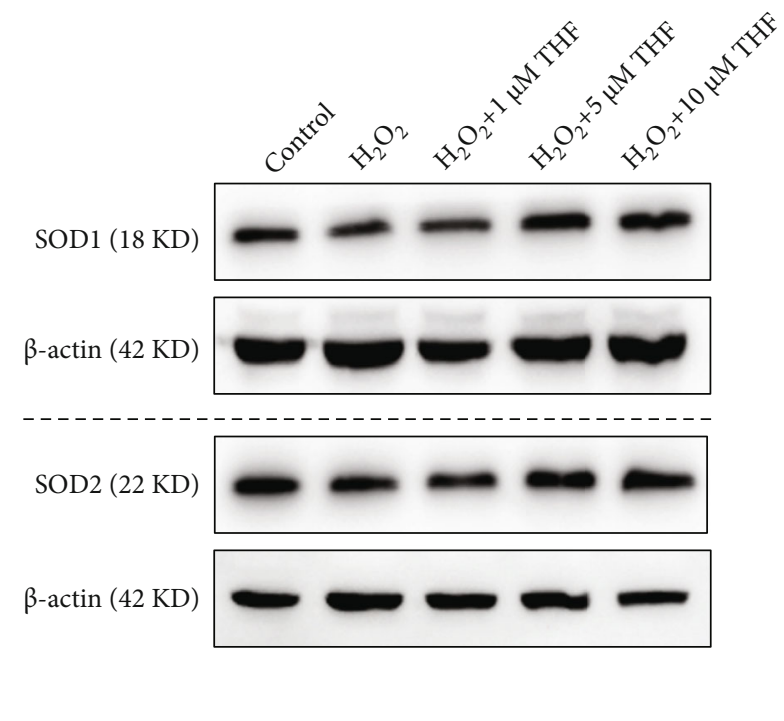

(c)

Figure 2: Continued. 


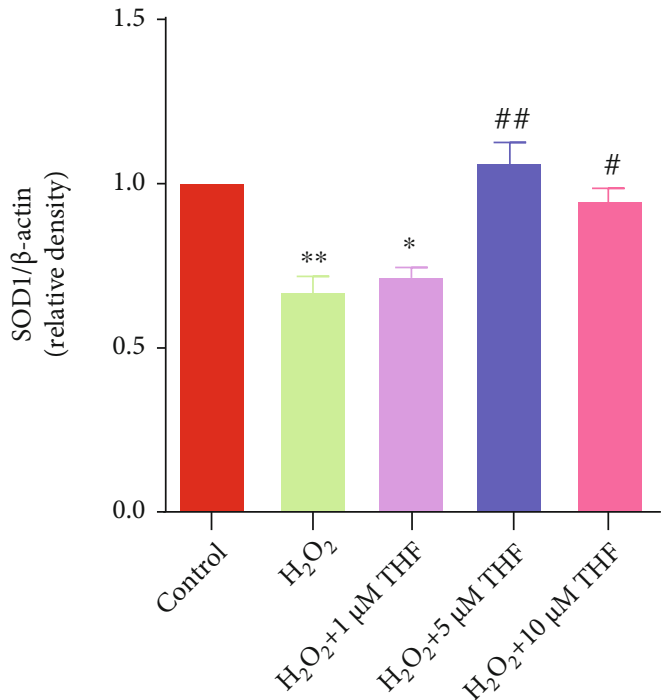

(d)

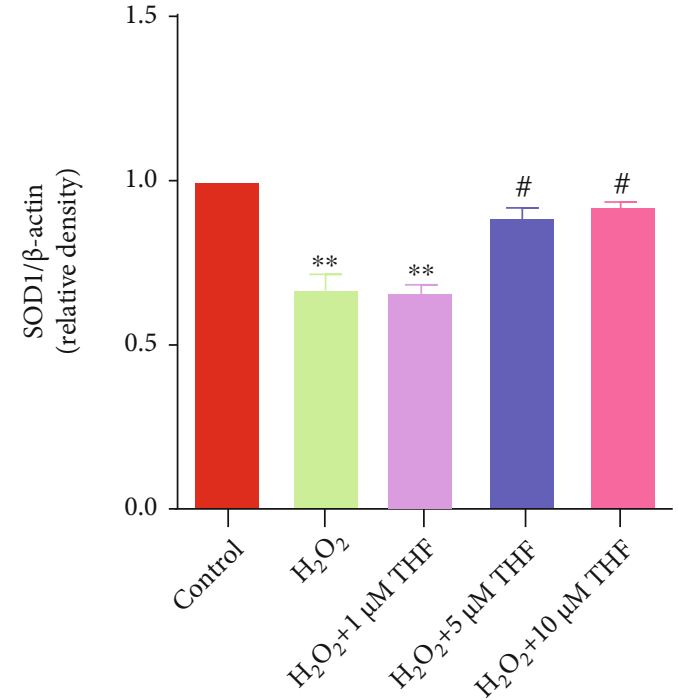

(e)

FIgURE 2: THF attenuated oxidative stress derived from $\mathrm{H}_{2} \mathrm{O}_{2}$ in NSC. (a) Mitochondrial ROS level in different groups. Scale bar: $10 \mu \mathrm{m}$. (b) Bar graph illustrating the optic intensity from (a). $N=6,{ }^{* * *} p<0.01$ vs. control group; ${ }^{*} p<0.05$ vs. $\mathrm{H}_{2} \mathrm{O}_{2}$ group. (c) Bands demonstrating the expression of SOD1 and SOD2 in each group. $\beta$-Actin was used as an internal control. (d) Semiquantitative analysis of SOD1 from (c). $N=3,{ }^{*} p<0.05,{ }^{* *} p<0.01$ vs. control group; ${ }^{\#} p<0.05,{ }^{\# \#} p<0.01$ vs. $\mathrm{H}_{2} \mathrm{O}_{2}$ group. (e) Semiquantitation of SOD2 from (c). $N=3,{ }^{*} p<0.05$, ${ }^{* *} p<0.01$ vs. control group; ${ }^{\#} p<0.05,{ }^{\# \#} p<0.01$ vs. $\mathrm{H}_{2} \mathrm{O}_{2}$ group.

Temecula, CA, USA). Then, specimens were blocked by $10 \%$ skimmed milk in TBST at room temperature for 2 hours. Afterward, they were incubated with rabbit anti-SOD1 (1:1000, CST, USA), rabbit anti-SOD2 (1:1000, CST, USA), rabbit anti-PTEN ( $1: 1000$, CST, USA), rabbit antiPhospho-Akt (1:1000, CST, USA), rabbit anti-Akt ( $1: 1000$, CST, USA), rabbit anti-Phospho-mTOR $(1: 1000$, CST, USA), rabbit anti-mTOR (1:1000, CST, USA), mouse anti-GAPDH (1:1000, Santa Cruz Biotechnology, CA, USA), or mouse anti- $\beta$-actin $(1: 1000$, Santa Cruz Biotechnology, CA, USA) antibodies overnight at $4^{\circ} \mathrm{C}$. Secondary anti-mouse and anti-rabbit horseradish peroxidase- (HRP-) conjugated antibodies (1:10000, Boster, Wuhan, China) were used to conjoin the primary antibodies at room temperature for 2 hours. Finally, blots were visualized by electrochemiluminescence (ECL) substrate (ThermoFisher, USA). Signals were detected by Image $\mathrm{Lab}^{\mathrm{TM}}$ software (Bio-Rad, California, USA) and analyzed by Image $\mathrm{Lab}^{\mathrm{TM}}$ software (Bio-Rad, California, USA).

2.11. Statistical Analysis. All data were expressed as the mean \pm S.D. and analyzed using SPSS 23.0 (SPSS Inc., Chicago, IL). Multiple comparisons were performed by oneway analysis of variance (ANOVA) and followed by Turkey's post hoc test if the data were normal distribution using a Shapiro-Wilk normality test. A $p<0.05$ was considered to be significantly different.

\section{Results}

3.1. Primary NSC Isolation and Characteristics. NSC were isolated from cerebral cortex of embryonic mice and cultured in floating condition. As shown in Figure 1(a), NSC were gradually formed from single cell to neurospheres after 48 hours. Then, most of the cultured cells exhibited Nestin ${ }^{+}$ and Sox $2^{+}$, which are two main NSC markers [38], using immunofluorescence (Figure 1(b)). Thereafter, the differentiating potency of the NSC was determined by immunofluorescence after cultured in differentiation medium for 7 days. The immunostaining images showed that the cultured NSC held the potency of differentiating into about $50.2 \%$ of $\mathrm{GFAP}^{+}$astrocytes, $36.8 \%$ of $\mathrm{MAP}^{+}$neurons, and $13 \%$ of Olig2 ${ }^{+}$oligodendrocytes (Figures $1(\mathrm{c})-1(\mathrm{f})$ ), indicating that the cultured NSC presented stemness and differentiating potential into astrocytes, neurons, and oligodendrocytes.

3.2. THF Attenuated Oxidative Stress Induced by $\mathrm{H}_{2} \mathrm{O}_{2}$ in NSC. To determine the effect of THF on NSC proliferation under oxidative stress condition, we firstly evaluated the effect of THF on the production of reactive oxygen species (ROS) in NSC. To introduce oxidative stress in NSC, $50 \mu \mathrm{M}$ hydrogen peroxide $\left(\mathrm{H}_{2} \mathrm{O}_{2}\right)$ was used. Meanwhile, three concentrations of THF $(1 \mu \mathrm{M}, 5 \mu \mathrm{M}$, and $10 \mu \mathrm{M})$ were used to evaluate the optimal working concentration for relieving oxidative stress. The results showed that, compared with control group, the fluorescence intensity of MitoROS in $\mathrm{H}_{2} \mathrm{O}_{2}$ group was obviously increased (Figures 2(a) and 2(b)). While this effect was evidently abrogated after treatment with $5 \mu \mathrm{M}$ and $10 \mu \mathrm{M}$ THF, no dramatic difference was observed in $1 \mu \mathrm{M}$ THF (Figures 2(a) and 2(b)). Furthermore, the expression level of SOD1 and SOD2 in $\mathrm{H}_{2} \mathrm{O}_{2}$ and $\mathrm{H}_{2} \mathrm{O}_{2}+1 \mu \mathrm{M}$ THF groups was significantly decreased, whereas this reduced expression level of SOD1 and SOD2 was partially reversed after application of $5 \mu \mathrm{M}$ and $10 \mu \mathrm{M}$ THF (Figures 2(c)-2(e)). These results indicated that the administration of $5 \mu \mathrm{M}$ and $10 \mu \mathrm{M}$ THF markedly 

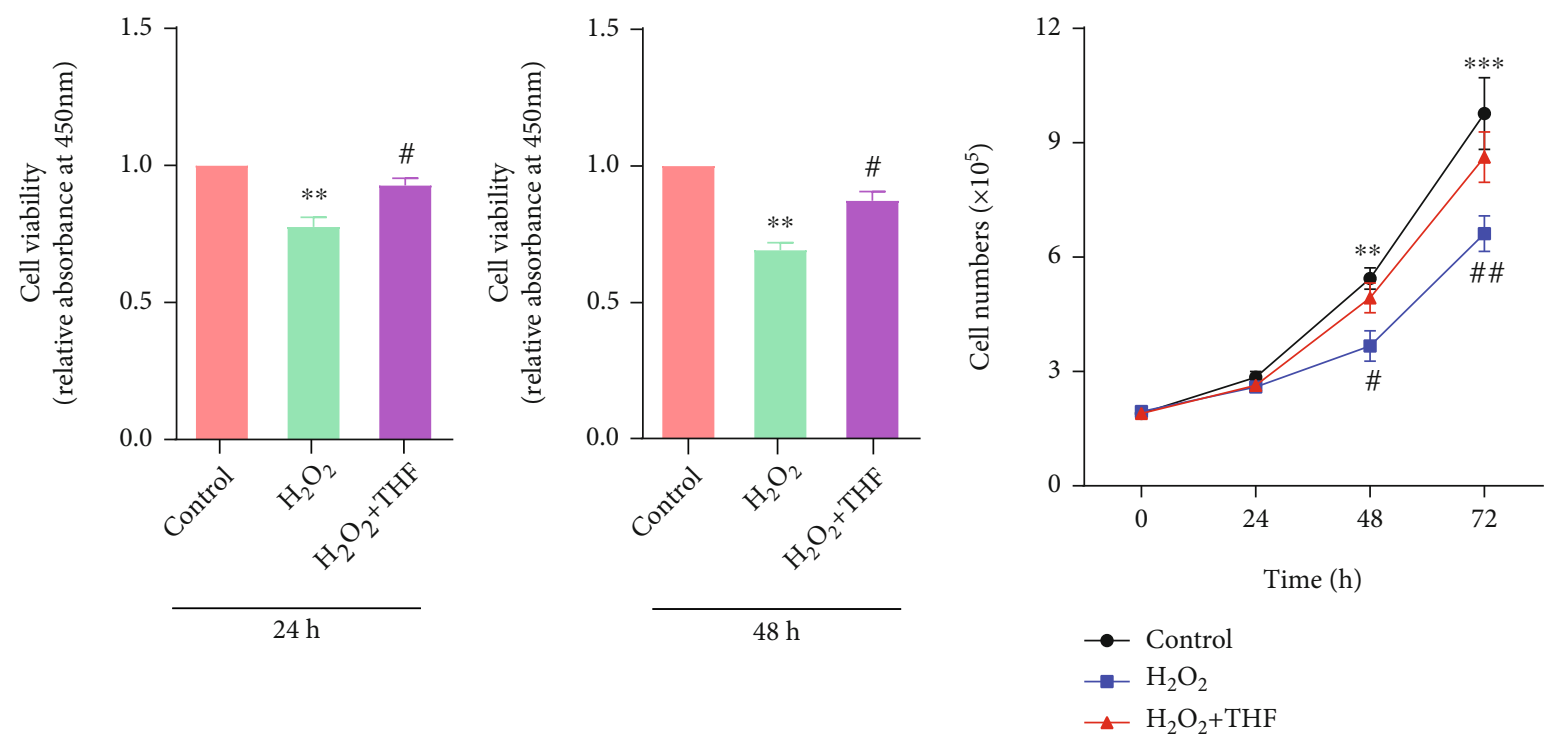

(a)
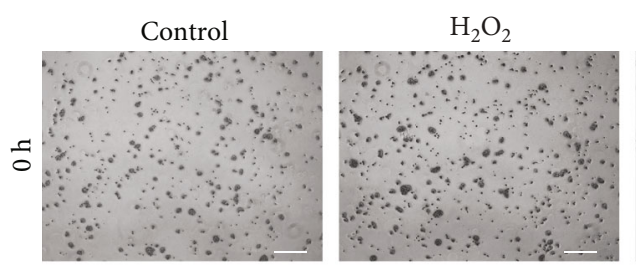

$\mathrm{H}_{2} \mathrm{O}_{2}+\mathrm{THF}$
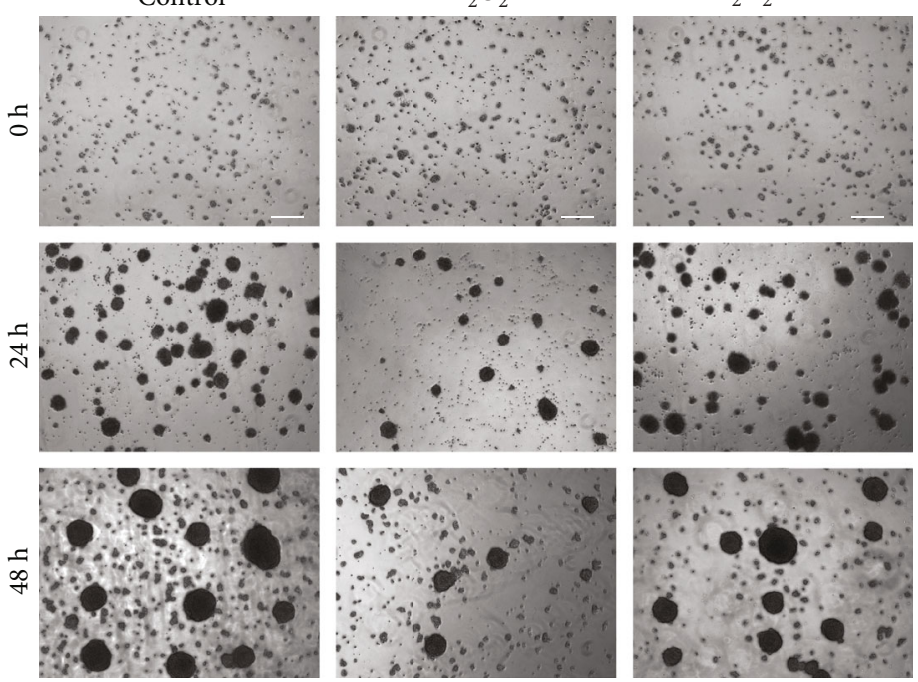

(c)

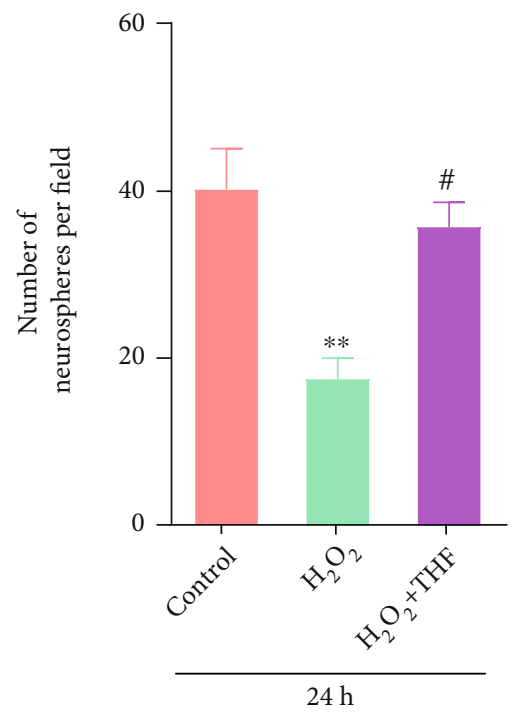

(d)
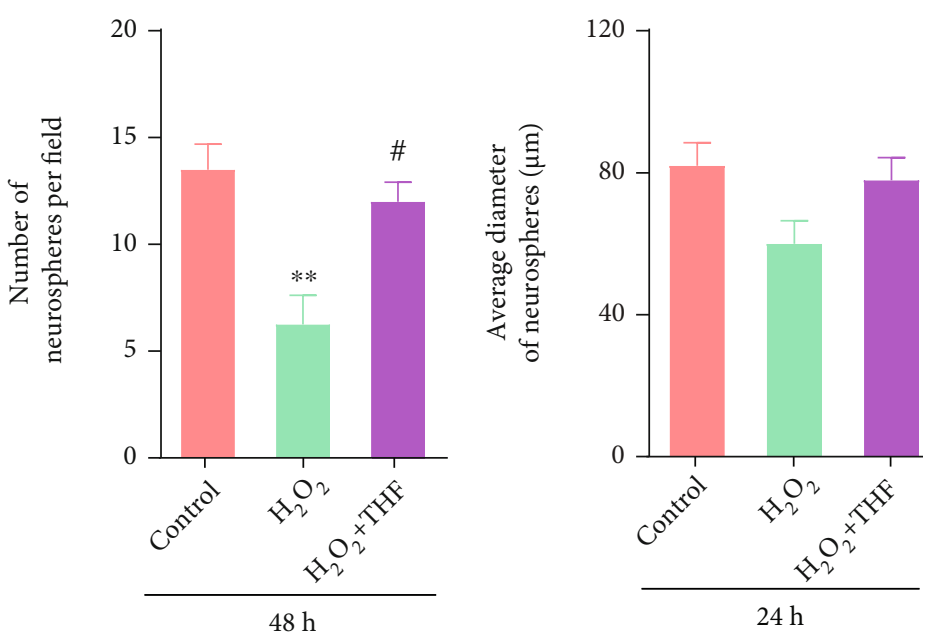

(e)

(f)

FIgURE 3: Continued. 


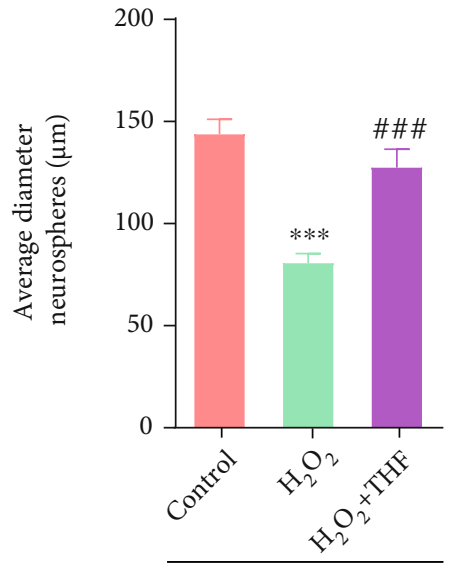

$48 \mathrm{~h}$
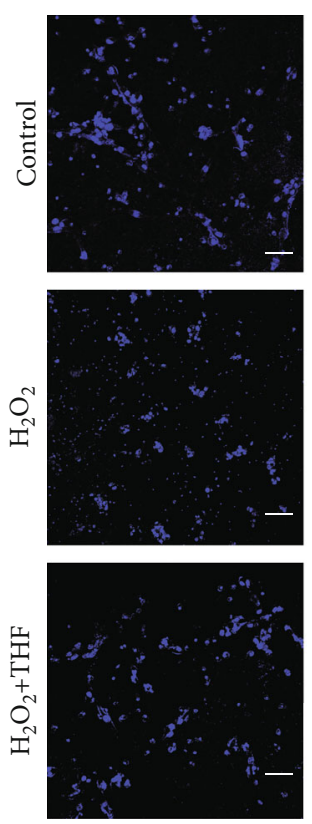

(g)
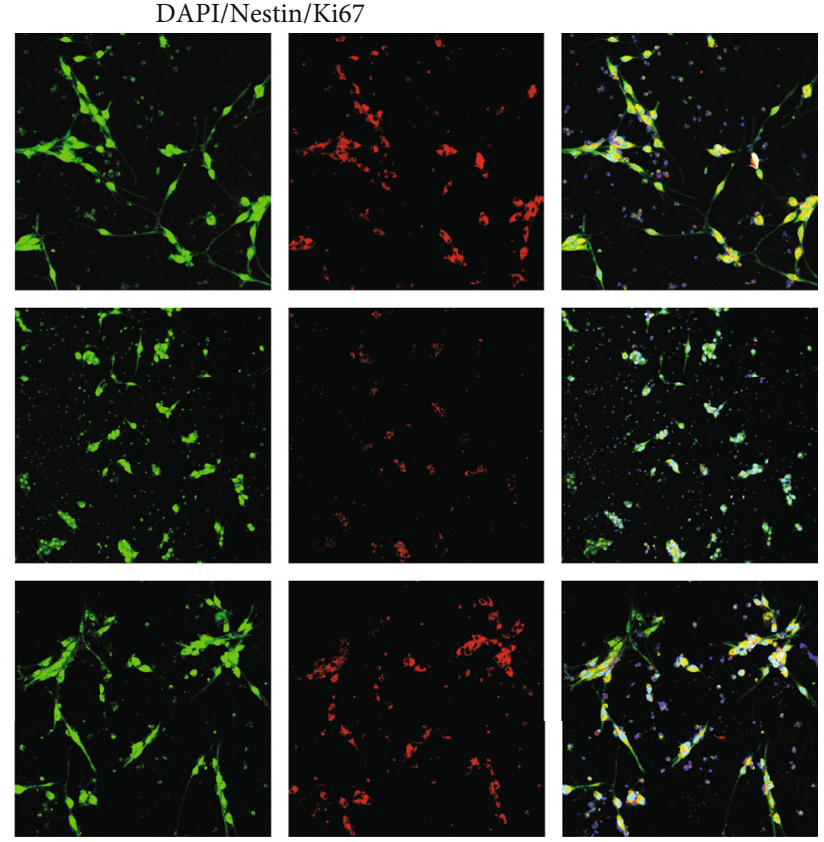

(h)

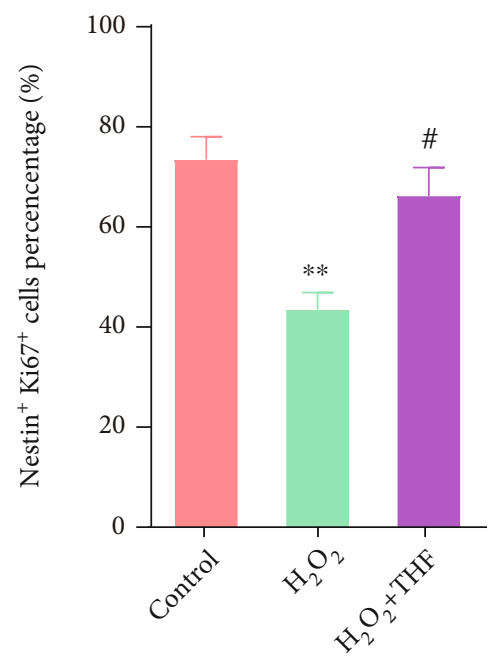

(i)

FIgURE 3: THF reversed the inhibitory effect of oxidative stress on NSC proliferation. (a) The absorbance value in each group at $24 \mathrm{~h}$ and 48 h using CCK-8 assays. $N=6,{ }^{* *} p<0.01$ vs. control group; ${ }^{*} p<0.05$ vs. $\mathrm{H}_{2} \mathrm{O}_{2}$ group. (b) Growth curve analysis in different groups. $N=4$, ${ }^{* *} p<0.01,{ }^{* * *} p<0.001$ vs. control group; ${ }^{\#} p<0.05,{ }^{\# \#} p<0.01$ vs. $\mathrm{H}_{2} \mathrm{O}_{2}$ group. (c) The phase-contrasted images depicting NSC condition in each group at different timepoints. Scale bars: $50 \mu \mathrm{m}$. (d) Bar graph summarizing the number of neurospheres per field among different groups at $24 \mathrm{~h} . N=5,{ }^{* *} p<0.01$ vs. control group; ${ }^{*} p<0.05$ vs. $\mathrm{H}_{2} \mathrm{O}_{2}$ group. (e) Bar chart representing the number of neurospheres per field among different groups at $48 \mathrm{~h} . \mathrm{N}=5,{ }^{* *} p<0.01$ vs. control group; ${ }^{*} p<0.05$ vs. $\mathrm{H}_{2} \mathrm{O}_{2}$ group. (f) Histogram illustrating the average diameter of neurospheres in each group at $24 \mathrm{~h}$. (g) Qualification of the average diameter of neurospheres in each group at $48 \mathrm{~h}$. $N=5$, ${ }^{* * *} p<0.001$ vs. control group; ${ }^{\# \#} p<0.001$ vs. $\mathrm{H}_{2} \mathrm{O}_{2}$ group. (h) The immunostaining of Nestin and Ki67 in various groups. Scale bars: $10 \mu \mathrm{m}$. (i) The statistical analysis of $\mathrm{Nestin}^{+} \mathrm{Ki} 7^{+}$from (h). $N=6,{ }^{* *} p<0.01$ vs. control group; ${ }^{*} p<0.05$ vs. $\mathrm{H}_{2} \mathrm{O}_{2}$ group.

attenuated oxidative stress, in comparison with that in control group. Therefore, $5 \mu \mathrm{M}$ THF was used in subsequent experiments in the present study.

3.3. THF Alleviated the Inhibitory Effect of Oxidative Stress on NSC Proliferation. To quantitatively assess the effect of THF on NSC proliferation under oxidative stress condition, CCK8 assays were firstly performed to elucidate cell viability in varying groups. The results showed that cell viability was significantly decreased when NSC were exposed to $\mathrm{H}_{2} \mathrm{O}_{2}$ for 24 hours and 48 hours (Figure 3(a)). Interestingly, cell viability was significantly increased when treated with $5 \mu \mathrm{M}$ THF compared with $\mathrm{H}_{2} \mathrm{O}_{2}$ group, but there was no significant difference between $\mathrm{H}_{2} \mathrm{O}_{2}+\mathrm{THF}$ group and control group (Figure 3(a)). Then, the growth curve analysis indicated that oxidative stress derived from $\mathrm{H}_{2} \mathrm{O}_{2}$ inhibited 


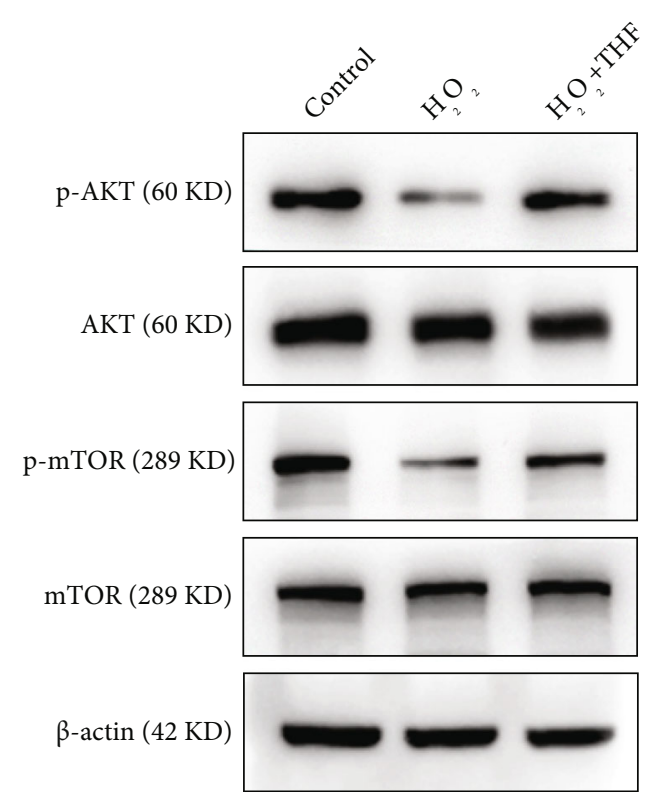

(a)

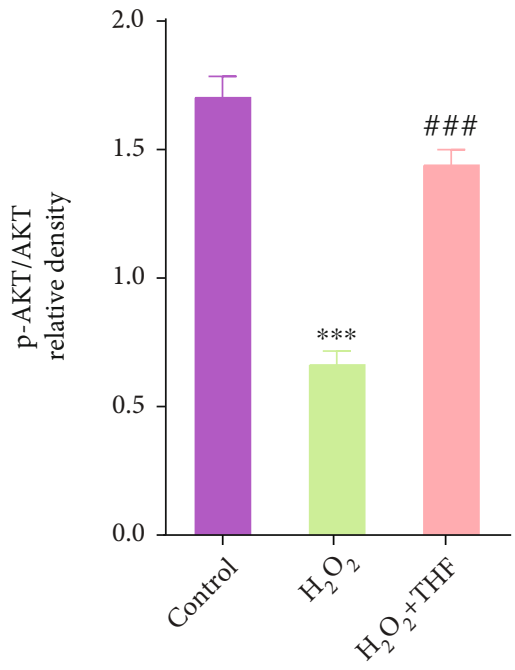

(b)

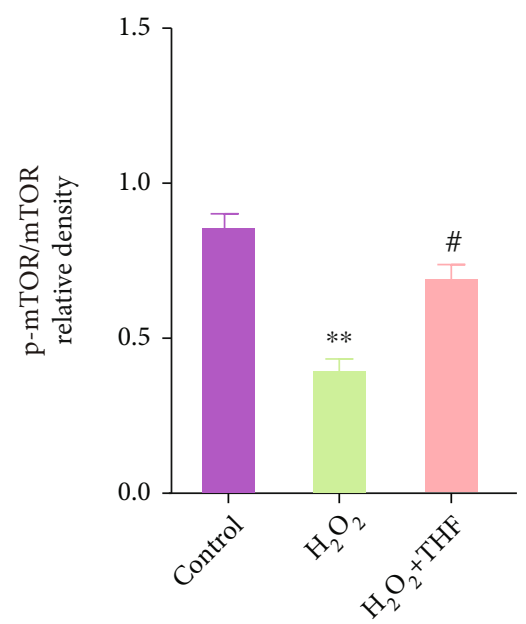

(c)

FIGURE 4: THF alleviated the inhibitory effect of oxidative stress on NSC proliferation through Akt/mTOR signaling pathway. (a) Bands depicted the expression of p-Akt, Akt, p-mTOR, and mTOR in different groups. (b) Semiquantitative analysis of p-Akt/Akt in control, $\mathrm{H}_{2} \mathrm{O}_{2}$, and $\mathrm{H}_{2} \mathrm{O}_{2}+$ THF groups from (a). $N=3$, ${ }^{* *} p<0.01$ vs. control group; ${ }^{*} p<0.05,{ }^{\# \#} p<0.01$ vs. $\mathrm{H}_{2} \mathrm{O}_{2}$ group. (c) Semiquantification of the expression of $\mathrm{p}-\mathrm{mTOR} / \mathrm{mTOR}$ from (a). $N=3,{ }^{* *} p<0.01$ vs. control group; ${ }^{\#} p<0.05,{ }^{\# \#} p<0.01$ vs. $\mathrm{H}_{2} \mathrm{O}_{2}$ group.

NSC proliferation, while THF partially restored the ability of NSC proliferation (Figure 3(b)). Furthermore, neurosphere formation assays demonstrated that oxidative stress reduced the number of neurospheres at 24 hours and 48 hours in $\mathrm{H}_{2} \mathrm{O}_{2}$ group (Figures 3(c)-3(e)). Meanwhile, the average diameter of neurospheres was obviously diminished in $\mathrm{H}_{2} \mathrm{O}_{2}$ group at 48 hours, while THF could alleviate this effect compared with control group (Figures $3(\mathrm{f})$ and $3(\mathrm{~g})$ ). In addition, the immunostaining of Nestin and Ki67 was applied to validate the effect of THF on NSC proliferation triggering by oxidative stress. The immunostaining images represented that the percentage of Nestin and Ki67 doublepositive cells in $\mathrm{H}_{2} \mathrm{O}_{2}$ group was surely lower than that in control group, and the proportion of Nestin and Ki67 double-positive cells was significantly higher in $\mathrm{H}_{2} \mathrm{O}_{2}+\mathrm{THF}$ group than that in $\mathrm{H}_{2} \mathrm{O}_{2}$ group (Figures $3(\mathrm{~h})$ and $3(\mathrm{i})$ ). Together, these results revealed that the administration of THF alleviated the inhibitory effect of oxidative stress on NSC proliferation.

3.4. THF Alleviated the Inhibitory Effect of Oxidative Stress on NSC Proliferation through PTEN/Akt/mTOR Signaling Pathway. Given that Akt/mTOR signaling pathway is a pivotal mediator for NSC expansion [30-33] and plays an important role in counteracting oxidative stress in macrophage and glioma cells [34-36], we then posited that Akt/ 


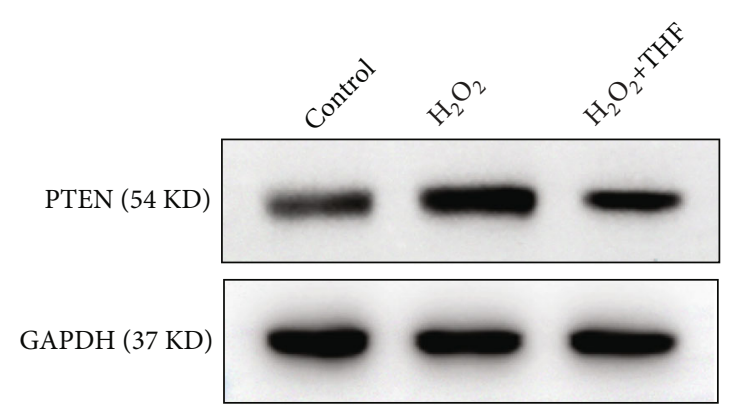

(a)

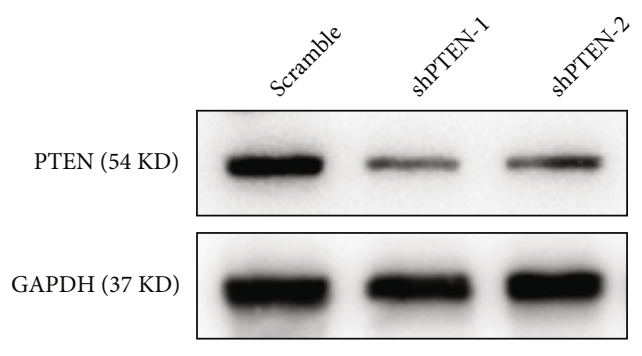

(c)

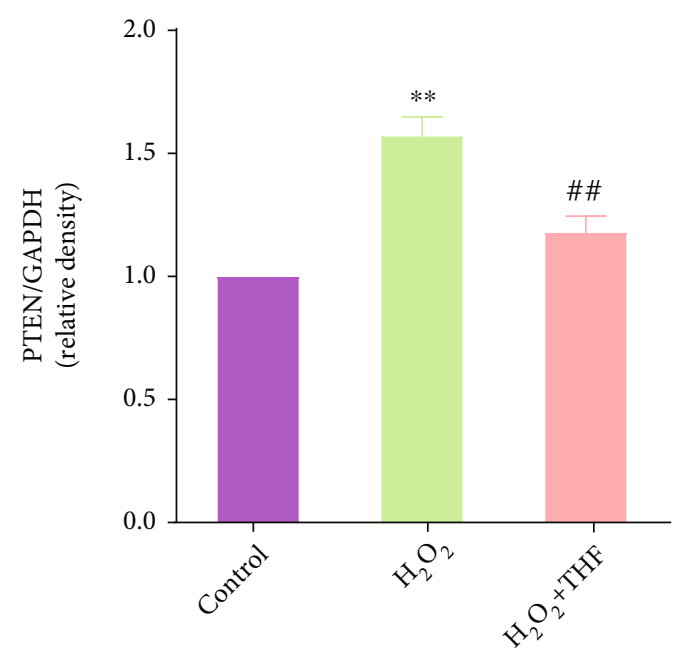

(b)

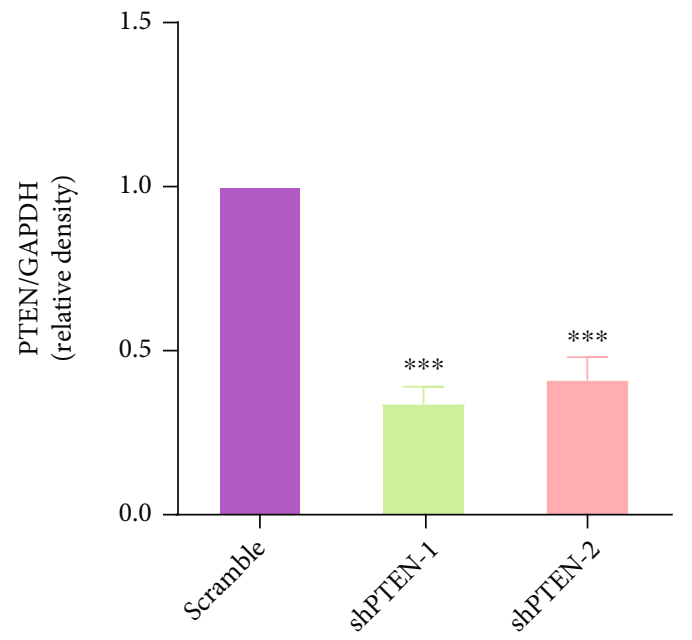

(d)

FIGURE 5: PTEN participated in THF partially abrogating the inhibitory effect induced by oxidative stress on NSC proliferation. (a) Immunoblot bands showing the expression of PTEN in control, $\mathrm{H}_{2} \mathrm{O}_{2}$, and $\mathrm{H}_{2} \mathrm{O}_{2}+$ THF groups. (b) Semiquantitative analysis of PTEN from (a). $N=3,{ }^{* *} p<0.01$ vs. control group; ${ }^{\# \#} p<0.01$ vs. $\mathrm{H}_{2} \mathrm{O}_{2}$ group. (c) Bands depicting the efficiency of PTEN KD in NSC. (d) Semiquantification from (c). $N=3,{ }^{* * *} p<0.001$ vs. scramble group.

mTOR signaling pathway was involved in regulating NSC proliferation resulting from oxidative stress. Herein, the expression of $\mathrm{p}$-Akt and $\mathrm{p}$-mTOR was determined using Western blotting. The bands depicted that the expression of p-Akt and p-mTOR was clearly downregulated in $\mathrm{H}_{2} \mathrm{O}_{2}$ group (Figures 4(a)-4(c)), while the reduced expression of p-Akt and p-mTOR was dramatically increased with the administration of $5 \mu \mathrm{M}$ THF (Figures $4(\mathrm{a})-4(\mathrm{c})$ ).

Considering that PTEN is the upstream effector of Akt/ mTOR signaling pathway and usually exerts neuroprotective effect on spiral ganglion neuron in response to oxidative stress [29], the expression of PTEN was assessed using Western blotting. Compared with control group, the expression of PTEN was significantly increased in $\mathrm{H}_{2} \mathrm{O}_{2}$ group (Figures 5(a) and 5(b)). To the contrary, the expression of PTEN in $\mathrm{H}_{2} \mathrm{O}_{2}+$ THF group was surely decreased (Figures 5(a) and 5(b)). Combined with the signaling pathway of Akt/mTOR aforementioned above, the results pre- liminarily certified the hypothesis that PTEN/Akt/mTOR signaling pathway played an evident role in the process that THF administration mitigated the inhibitory effect of oxidative stress on NSC proliferation.

To further decipher the role of PTEN/Akt/mTOR signaling pathway in refreshing NSC proliferation potential that was suppressed by oxidative stress, shPTEN lentivirus was used to knock down the expression of PTEN in NSC. The expression of PTEN was markedly diminished with the application of shPTEN-1 and shPTEN-2 lentivirus (Figures 5(c) and 5(d)). Given that the higher efficiency of shPTEN-1 lentivirus, the shPTEN-1 lentivirus was utilized in our future experiments. Then, the effect of PTEN KD on oxidative stress was investigated. The results presented that, compared with scramble group, the fluorescence intensity of MitoROS in $\mathrm{H}_{2} \mathrm{O}_{2}$ group was obviously increased, while this effect was dramatically abolished with application of $5 \mu \mathrm{M}$ THF and/or shPTEN lentivirus (Figures 6(a) and 


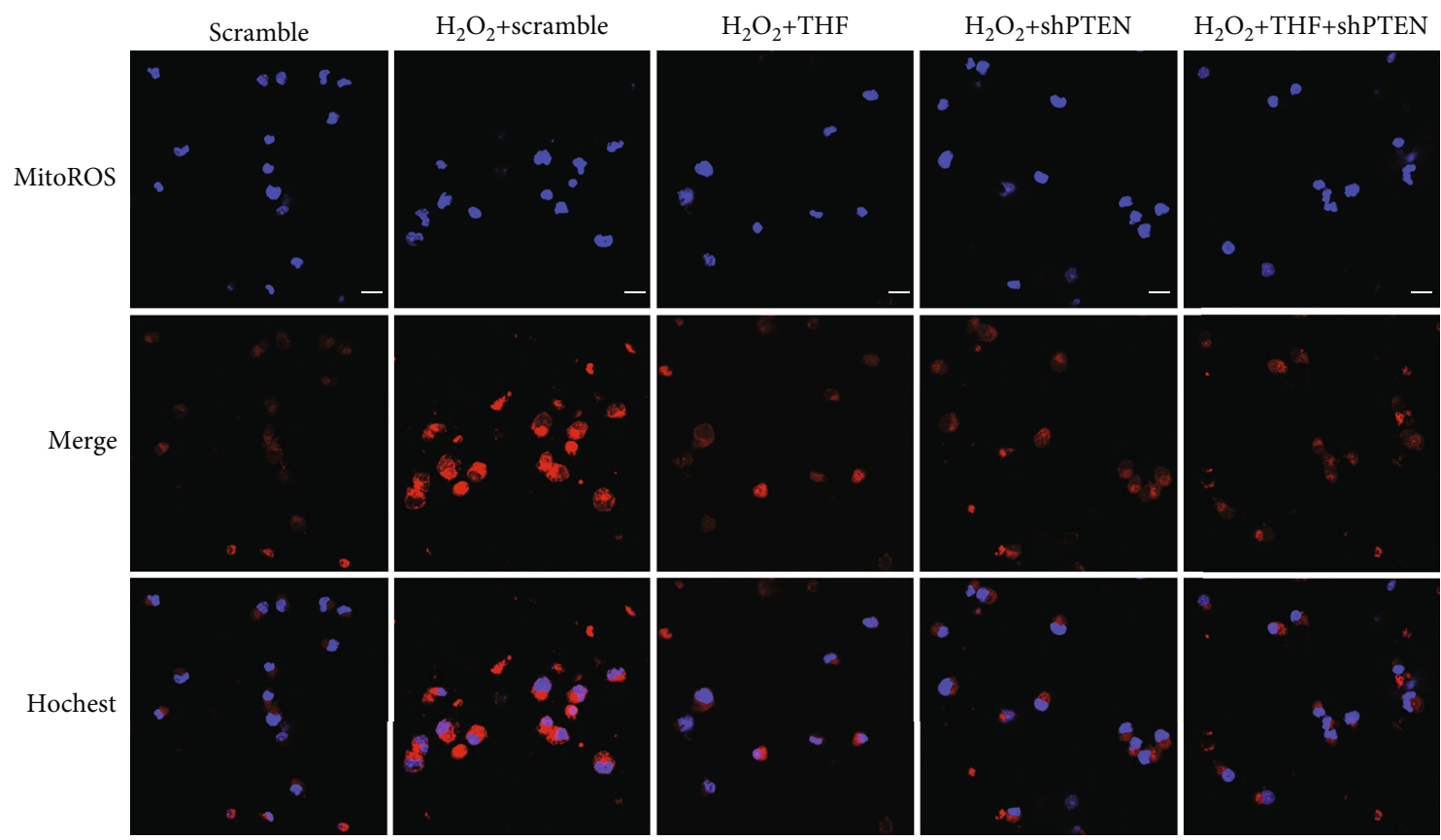

(a)

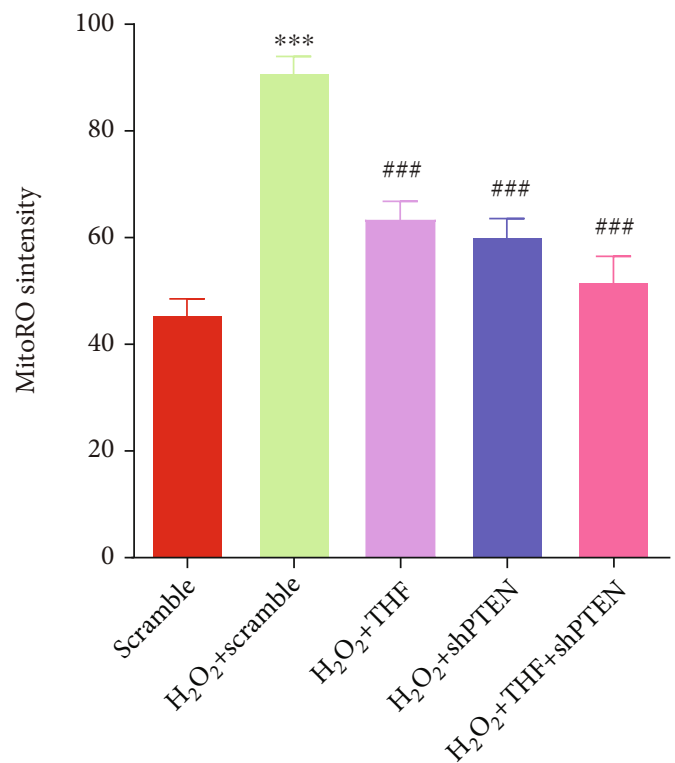

(b)
SOD1 (18 KD)

$\beta$-actin (42 KD)

SOD2 (22 KD)

$\beta$-actin (42 KD)

$$
\mathrm{H}_{2} \mathrm{O}
$$$$
\text { THF }
$$

ShPTEN 


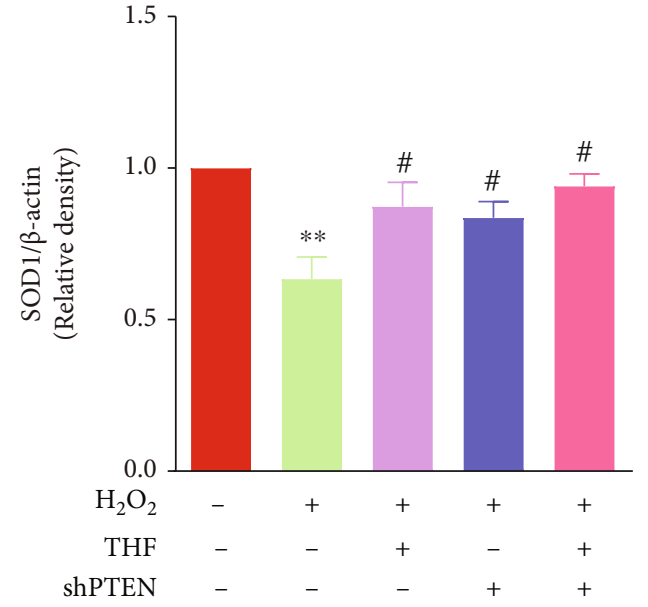

(d)

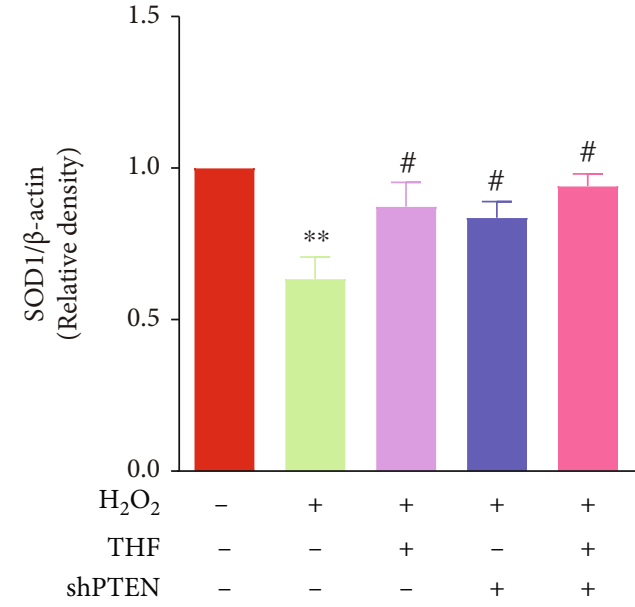

(e)

FIgURE 6: PTEN KD played the same role as THF in reducing oxidative stress in NSC. (a) Mitochondrial ROS level in different groups. Scale bar: $10 \mu \mathrm{m}$. (b) Bar graph illustrating the optic intensity from (a). $N=4,{ }^{* * *} p<0.001$ vs. scramble group; ${ }^{\# \# \#} p<0.001$ vs. scramble $+\mathrm{H}_{2} \mathrm{O}_{2}$ group. (c) Bands demonstrating the expression of SOD1 and SOD2 in each group. $\beta$-Actin was used as a loading control. (d) Semiquantitation of SOD1 from (c). $N=3,{ }^{* *} p<0.01$ vs. scramble group; ${ }^{*} p<0.05$ vs. scramble $+\mathrm{H}_{2} \mathrm{O}_{2}$ group. (e) Semiquantification of SOD2 from (c). $N=3,{ }^{* *} p<0.01$ vs. scramble group; ${ }^{\#} p<0.05,{ }^{\# \#} p<0.01$ vs. scramble $+\mathrm{H}_{2} \mathrm{O}_{2}$ group.

6(b)). Furthermore, the expression level of SOD1 and SOD2 in $\mathrm{H}_{2} \mathrm{O}_{2}$ group was obviously reduced, whereas this decreased expression level of SOD1 and SOD2 was abolished with the administration of $5 \mu \mathrm{M}$ THF and/or shPTEN lentivirus (Figures 6(c)-6(e)), to some extent. These results demonstrated that PTEN KD lentivirus exerted almost the same effect as $5 \mu \mathrm{M}$ THF in eliminating oxidative stress.

Afterward, the effect of PTEN KD on NSC proliferation was investigated. Firstly, the CCK8 assays demonstrated that the absorbance value in scramble, $\mathrm{H}_{2} \mathrm{O}_{2}+\mathrm{THF}, \mathrm{H}_{2} \mathrm{O}_{2}+$ shPTEN, and $\mathrm{H}_{2} \mathrm{O}_{2}+\mathrm{THF}+$ shPTEN groups was higher than that in scramble $+\mathrm{H}_{2} \mathrm{O}_{2}$ group at 24 hours and 48 hours (Figures $7(\mathrm{a})$ and $7(\mathrm{~b})$ ). Meanwhile, the growth curve analysis indicated that PTEN KD restored proliferation ability of NSC subjected to oxidative stress, in a degree (Figure 7(c)). Further, neurosphere formation assays indicated that the number of neurospheres at 24 hours and 48 hours and the diameter at 48 hours in scramble, $\mathrm{H}_{2} \mathrm{O}_{2}+\mathrm{THF}, \mathrm{H}_{2} \mathrm{O}_{2}+$ shPTEN, and $\mathrm{H}_{2} \mathrm{O}_{2}+\mathrm{THF}+$ shPTEN groups were profoundly increased compared with scramble $+\mathrm{H}_{2} \mathrm{O}_{2}$ group, while no significant difference was observed among scramble group, $\mathrm{H}_{2} \mathrm{O}_{2}+\mathrm{THF}$ group, $\mathrm{H}_{2} \mathrm{O}_{2}+$ shPTEN group, and $\mathrm{H}_{2} \mathrm{O}_{2}+\mathrm{THF}+$ shPTEN group (Figures $8(\mathrm{a})-8(\mathrm{e})$ ). Additionally, the immunostaining of Nestin and Ki67 was applied to elucidate the role of THF in NSC proliferation initiated by oxidative stress. The immunostaining images depicted that the percentage of Nestin and Ki67 doublepositive cells in $\mathrm{H}_{2} \mathrm{O}_{2}$ group was surely lower than that in scramble group, and the proportion of Nestin and Ki67 double-positive cells was significantly higher in $\mathrm{H}_{2} \mathrm{O}_{2}+$ THF, $\mathrm{H}_{2} \mathrm{O}_{2}+$ shPTEN, and $\mathrm{H}_{2} \mathrm{O}_{2}+\mathrm{THF}+$ shPTEN groups, while no marked significance was observed among scramble group, $\mathrm{H}_{2} \mathrm{O}_{2}+$ THF group, $\mathrm{H}_{2} \mathrm{O}_{2}+$ shPTEN group, and $\mathrm{H}_{2} \mathrm{O}_{2}+\mathrm{THF}+$ shPTEN group (Figure $8(\mathrm{f})$ ).
Collectively, these results demonstrated that administration of PTEN lentivirus alleviated the inhibitory effect on NSC expansion resulted from oxidative stress.

Subsequently, the downstream effectors of Akt and mTOR were determined to test the role of PTEN/Akt/ mTOR signaling pathway in regaining NSC proliferation potential attenuated by oxidative stress using Western blotting. The immunoblot bands illustrated that the reduced expression of p-Akt in $\mathrm{H}_{2} \mathrm{O}_{2}$ group was remarkably enhanced in $\mathrm{H}_{2} \mathrm{O}_{2}+$ THF, $\mathrm{H}_{2} \mathrm{O}_{2}+$ shPTEN, and $\mathrm{H}_{2} \mathrm{O}_{2}+\mathrm{THF}+$ shPTEN groups, while no marked significance was observed among scramble group, $\mathrm{H}_{2} \mathrm{O}_{2}+$ THF group, $\mathrm{H}_{2} \mathrm{O}_{2}+$ shPTEN group, and $\mathrm{H}_{2} \mathrm{O}_{2}+\mathrm{THF}+$ shPTEN group (Figures 9(a) and 9(b)). Meanwhile, the expression of p-mTOR demonstrated the same tendency as p-Akt among various groups (Figures 9(c) and 9(d)). Collectively, these results showcased that THF refreshed the restricted proliferating capacity of NSC under oxidative stress condition through PTEN/Akt/mTOR signaling pathway.

\section{Discussion}

In the present study, the results demonstrated that oxidative stress suppressed NSC proliferation, which was significantly alleviated by THF administration $(5 \mu \mathrm{M}$ and $10 \mu \mathrm{M})$. A negative relationship was found between PTEN expression and p-Akt or p-mTOR expression. Further evidence revealed that THF administration reduced the inhibitory effect of oxidative stress on NSC proliferation ascribing to activating the PTEN/Akt/mTOR signaling pathway.

The PTEN/Akt/mTOR signaling pathway is usually activated to exert multiple biological effects such as antioxidation when the ROS level is too high to beyond the physiologically antioxidative capability. Here, the results demonstrated that 


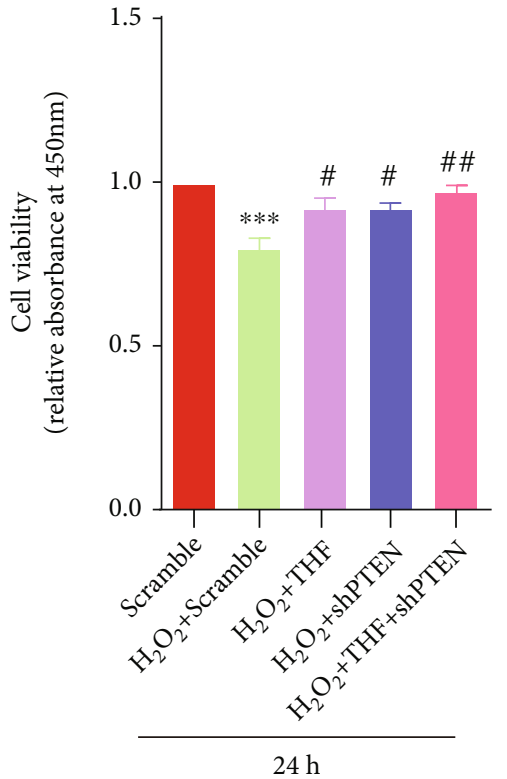

(a)

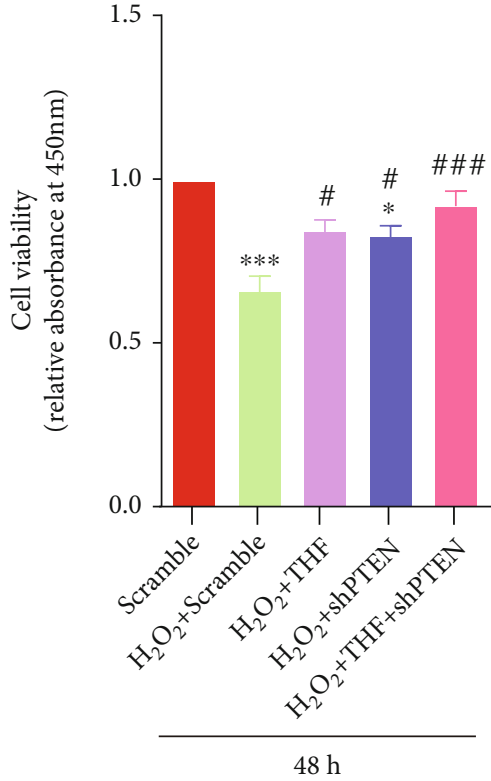

(b)

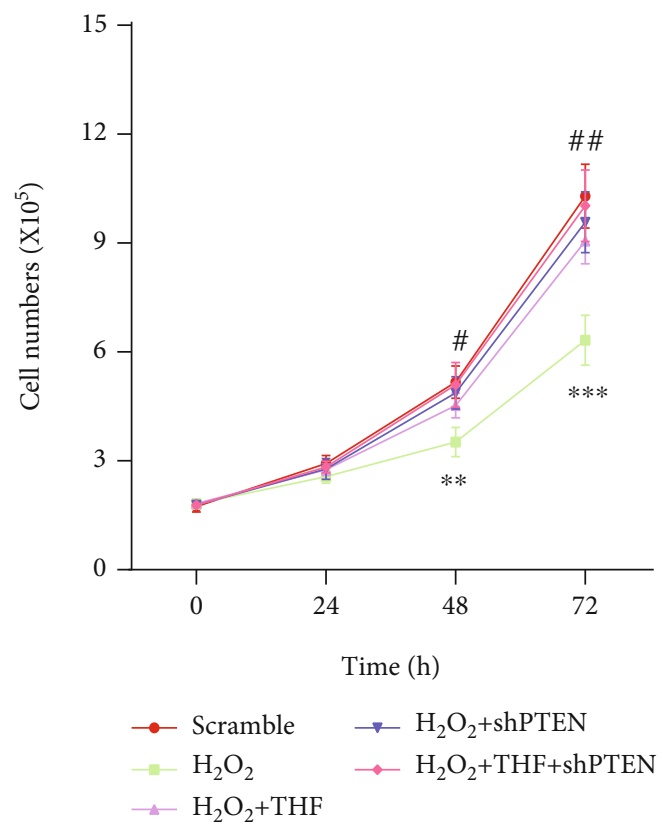

(c)

FIGURE 7: PTEN KD regained the inhibitory NSC proliferation capacity resulting from oxidative stress. (a) Bar graph summarizing the proliferation index of NSC using CCK8 at $24 \mathrm{~h}$ in each group. $N=6,{ }^{* * *} p<0.001$ vs. scramble group; ${ }^{\#} p<0.05,{ }^{\# \#} p<0.01$ vs. scramble + $\mathrm{H}_{2} \mathrm{O}_{2}$ group. (b) Histogram demonstrating the proliferation indice of NSC at $48 \mathrm{~h}$ using CCK8 in varying groups. $N=6,{ }^{*} p<0.05,{ }^{* * *} p$ $<0.001$ vs. scramble group; ${ }^{\#} p<0.05,{ }^{\# \# \#} p<0.001$ vs. scramble $+\mathrm{H}_{2} \mathrm{O}_{2}$ group. (c) Growth curve analysis in different groups. $N=5$, ${ }^{* *} p$ $<0.01,{ }^{* * *} p<0.001$ vs. scramble group; ${ }^{\#} p<0.05$, ${ }^{\# \#} p<0.01$ vs. scramble $+\mathrm{H}_{2} \mathrm{O}_{2}$ group.

a high ROS level induced by $50 \mu \mathrm{M} \mathrm{H}_{2} \mathrm{O}_{2}$ possessed the ability of increasing the expression of PTEN, while reducing the expression of $\mathrm{p}$-Akt and $\mathrm{p}-\mathrm{mTOR}$, indicating that PTEN negatively mediates the Akt/mTOR signaling pathway, which is consistent with previous studies [39, 40]. Meanwhile, the results delineated that the expression of PTEN was upregulated when primary NSC were subjected to oxidative stress, which exists a discrepancy with previous studies [39, 40]. The reason for this phenomenon might be ascribed to the cell subtype difference as these researches focus on the terminally differentiated somatic cells such as mesothelial cells and retinal pigment epithelium (RPE) cells, instead of stem cells, which bear the potency of differentiating into terminally differentiated somatic cells [41].

NSC, one subpopulation of stem cells, maintain a homeostasis among self-renewal, differentiation, and quiescence under physiological condition [5], whereas NSC possess the capacity of proliferating in situ, migrating toward 


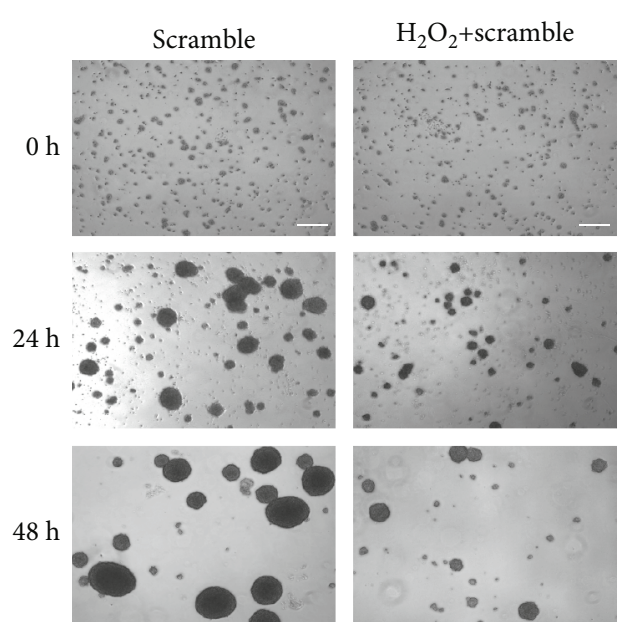

$24 \mathrm{~h}$

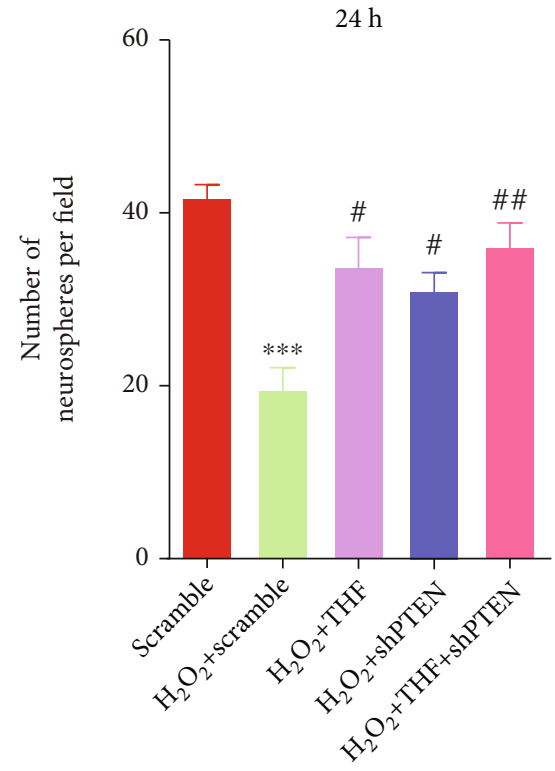

(b)

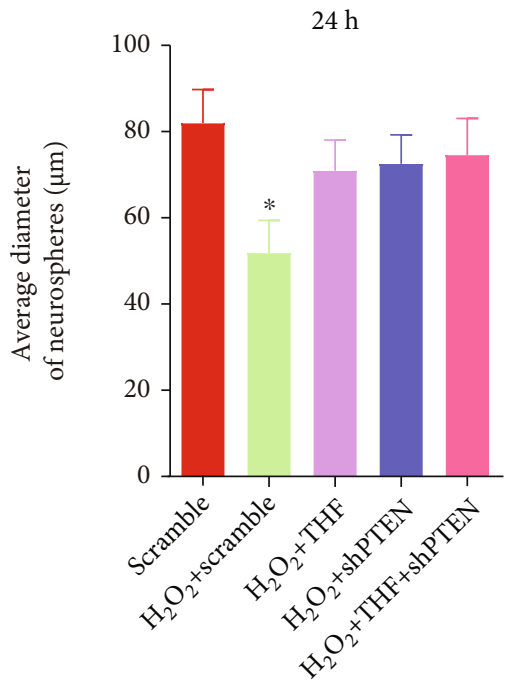

(d)

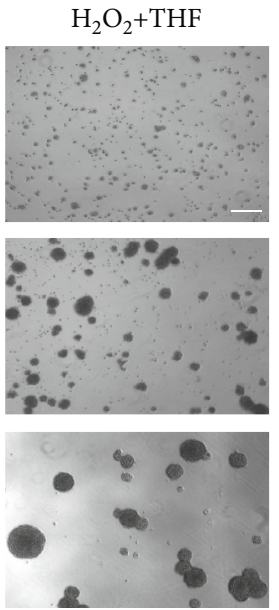

(a)
$\mathrm{H}_{2} \mathrm{O}_{2}+$ shPTEN $\quad \mathrm{H}_{2} \mathrm{O}_{2}+$ THF + shPTEN
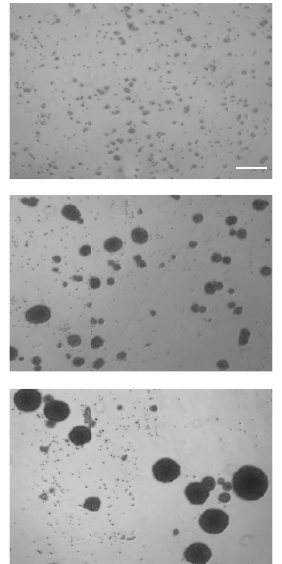

$48 \mathrm{~h}$

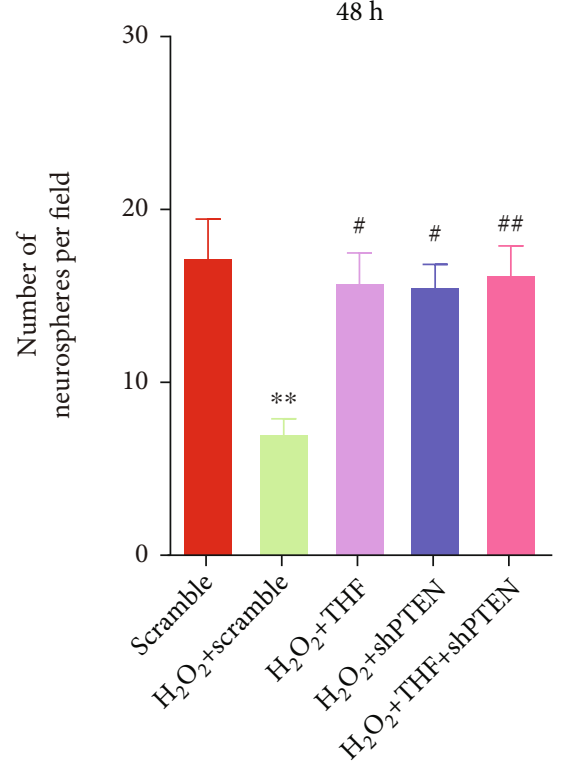

(c)

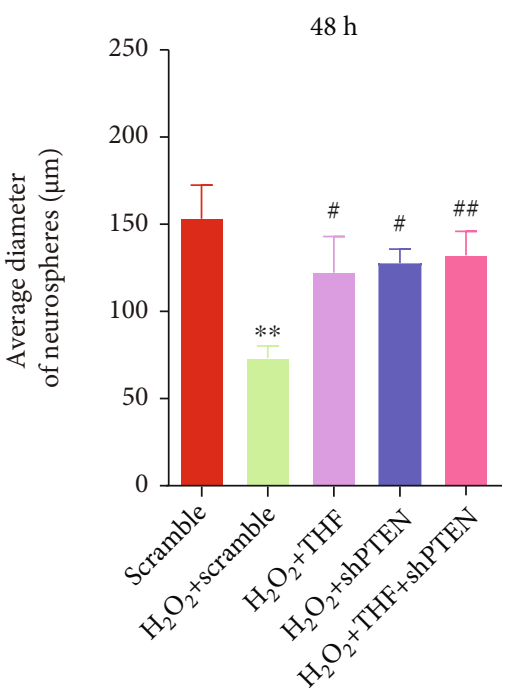

(e)

Figure 8: Continued. 

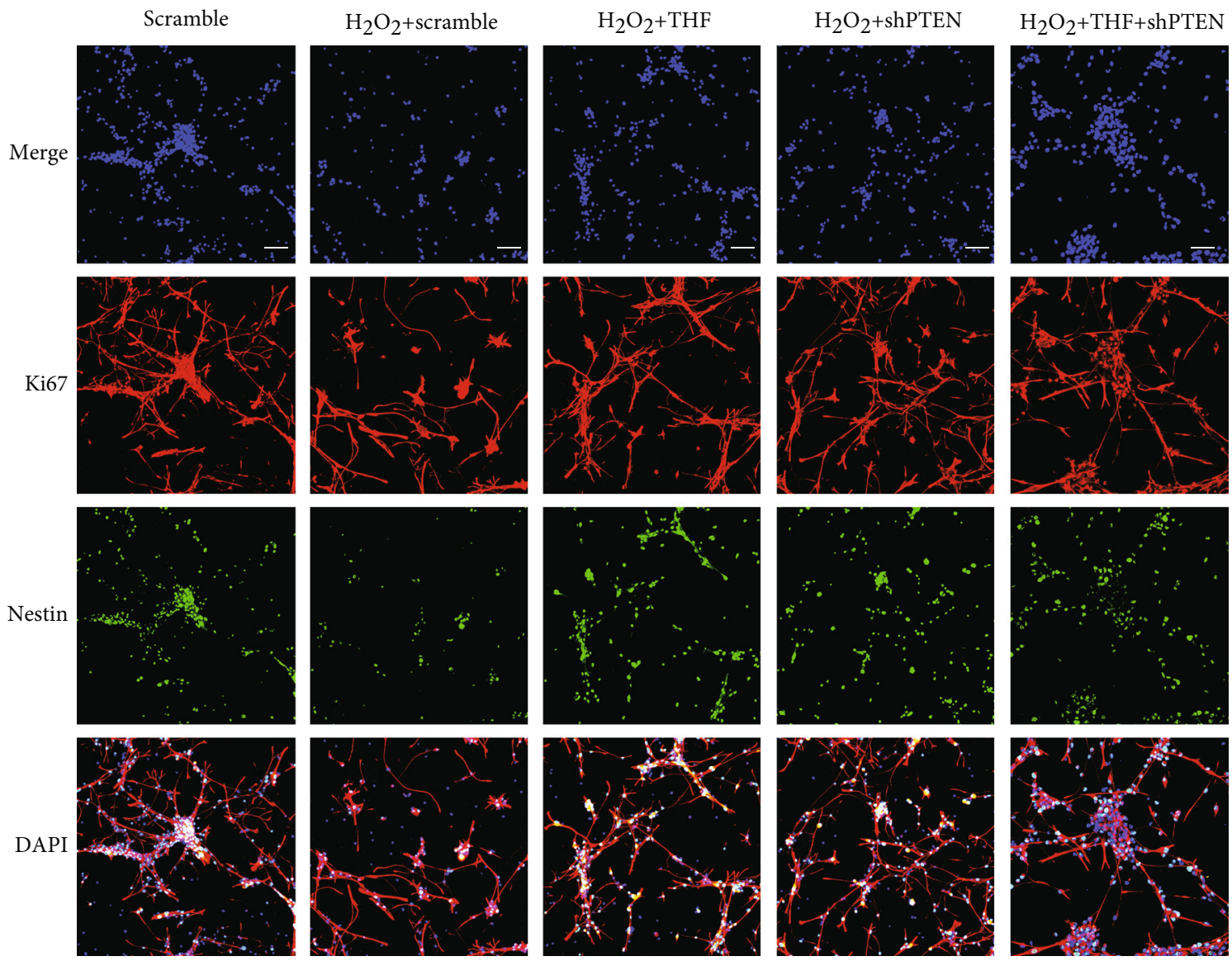

$(\mathrm{f})$

FIGURE 8: PTEN KD exerted the same effect as THF on retrieving NSC proliferation potential suppressed by oxidative stress. (a) The phasecontrasted images depicting NSC growth in each group at different timepoints. Scale bars: $50 \mu \mathrm{m}$. (b) Bar graph summarizing the number of neurospheres per field among different groups at $24 \mathrm{~h} . \mathrm{N}=5,{ }^{* * *} p<0.001$ vs. scramble group; ${ }^{*} p<0.05,{ }^{\# \#} p<0.01$ vs. scramble $+\mathrm{H}_{2} \mathrm{O}_{2}$ group. (c) Bar chart demonstrating the number of neurospheres per field among different groups at $48 \mathrm{~h} . N=5,{ }^{* *} p<0.01 \mathrm{vs}$. scramble group; ${ }^{\#} p<0.05,{ }^{\# \#} p<0.01$ vs. scramble $+\mathrm{H}_{2} \mathrm{O}_{2}$ group. (d) Histogram illustrating the average diameter of neurospheres in each group at $24 \mathrm{~h} . N=5,{ }^{*} p<0.5$ vs. scramble group. (e) Qualification of the average diameter of neurospheres in each group at $48 \mathrm{~h} . N=5,{ }^{* *} p<$ 0.01 vs. scramble group; ${ }^{\#} p<0.05,{ }^{\# \#} p<0.01$ vs. scramble $+\mathrm{H}_{2} \mathrm{O}_{2}$ group. (f) The immunostaining of Nestin and Ki67 in various groups. Scale bars: $50 \mu \mathrm{m}$.

the injured regions, differentiating into three main neural subpopulations (neurons, astrocytes, and oligodendrocytes), and integrating into the damaged neurovascular network to initiate neuro-regenerative ability after CNS injury [6-9]. Our previous studies have identified some factors inhibiting the regenerative ability of NSC, such as actin alpha 2 (ACTA2) [8], G protein-coupled estrogen receptor 1 (GPER1) [2], and chondroitin sulfate proteoglycan (CSPG) [42]. Meanwhile, a few therapeutic strategies are developed to strengthen the rehabilitative potential of NSC, such as the ascorbic acid (AA) receptor-sodium-vitamin $\mathrm{C}$ cotransporter 2 (SVCT2), poly-L-ornithine (PO) [9, 38], ambroxol [7], repetitive transcranial magnetic stimulation (rTMS) [43], and 15d-PGJ2, an endogenous ligand of peroxisome proliferator-activated receptor $\gamma(\operatorname{PPAR} \gamma)$ [44]. In the present study, THF is proven to be an effective treatment recovering the inhibitory proliferation capacity of NSC resulting from oxidative stress.
Oxidative stress is a common pathology in varying CNS diseases. Previous studies have indicated that oxidative stress results in lipid peroxidation which leads to neural cells apoptosis in the cerebellum, hippocampus, and cerebral cortex after TBI $[15,16]$, cerebral stroke [17-19], and neurodegenerative diseases [20-22]. Herein, reducing oxidative stress is an effective approach to diminish loss of neural cells and enhance neuro-regenerative capacity. Here, the results certified that oxidative stress inhibited NSC proliferation to hinder the rehabilitative ability after CNS injury. Furthermore, THF has been validated to be a promising treatment in alleviating oxidative stress, therefore, recalling the inhibitory effect of oxidative stress on NSC proliferation.

THF, a crucial cofactor for the conversion of homocysteine to methionine, holds multifaced neuroprotective effects after CNS injury. Hyperhomocysteinemia, characterized as the elevated plasma levels of homocysteine, has been showed to be a risk factor for neurodevelopmental and 


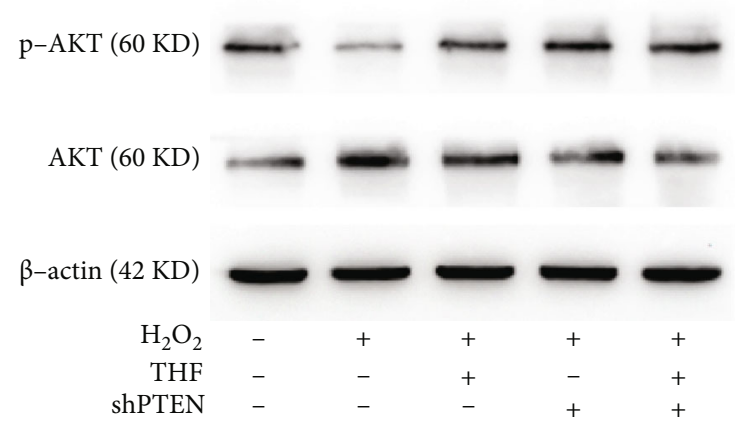

(a)

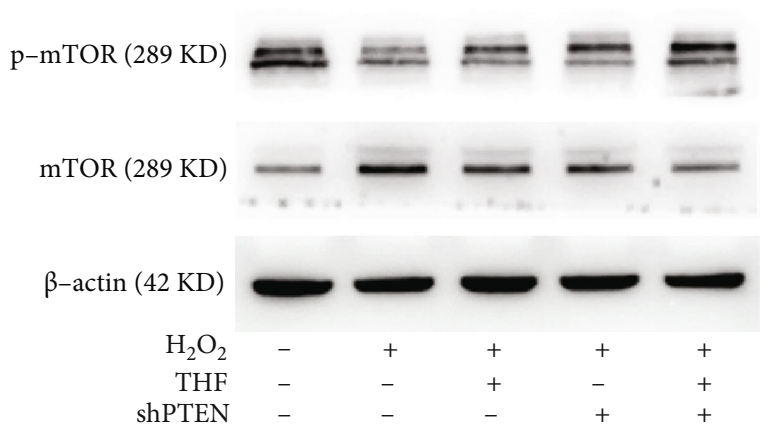

(c)

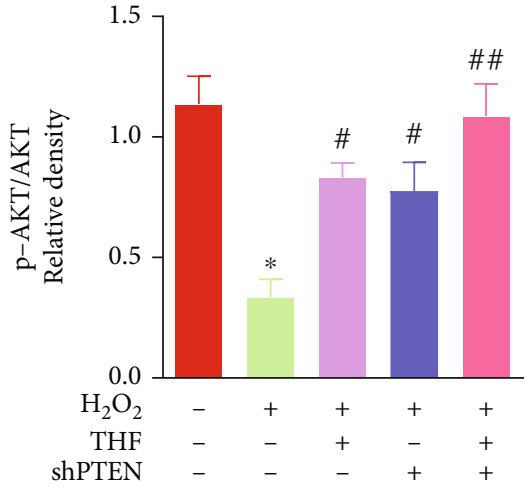

(b)

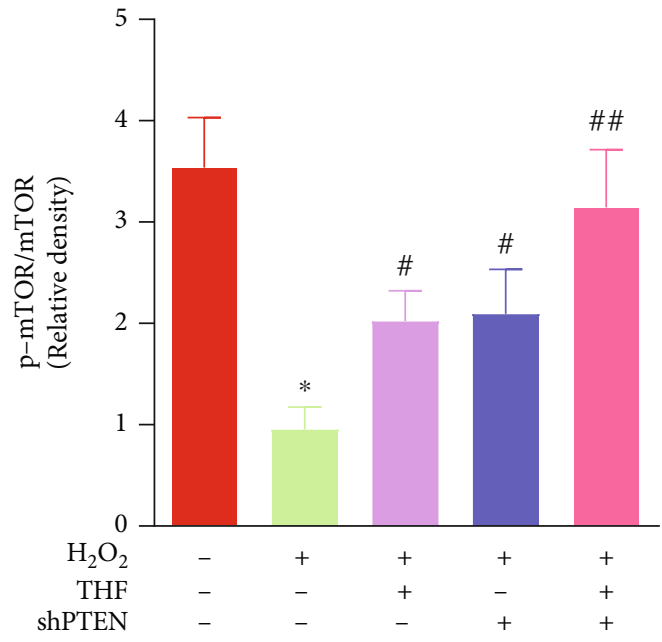

(d)

FIGURE 9: PTEN/Akt/mTOR signaling pathway played an evident role in THF mitigating the reduced NSC proliferation ability initiated by oxidative stress. (a) Immunoblot bands illustrating the expression of p-Akt and Akt in different groups. (b) Semiquantitative analysis of pAkt/Akt from (a). $N=3,{ }^{*} p<0.05,{ }^{* *} p<0.01$ vs. scramble group; ${ }^{\#} p<0.05,{ }^{\# \#} p<0.01$ vs. scramble $+\mathrm{H}_{2} \mathrm{O}_{2}$ group. (c) Bands demonstrating the expression of p-mTOR and mTOR in each group. (d) Semiquantification of p-mTOR/mTOR from (c). $N=3,{ }^{*} p<0.05,{ }^{* *} p<0.01$ vs. scramble group; ${ }^{\#} p<0.05,{ }^{\# \#} p<0.01$ vs. scramble $+\mathrm{H}_{2} \mathrm{O}_{2}$ group.

neurodegenerative disorders. Research has demonstrated that THF application clearly reduces the increased level of homocysteine in plasma to decrease ROS production, thereafter, decreasing the apoptosis of neural cells [45]. Meantime, previous investigations have showcased that ROS accumulation initiates immune response which causes damage to neural cells after CNS injury [46, 47]. Furthermore, our previous report also shows that ROS accumulation triggers ferroptosis, and ferrostatin-1, a potent inhibitor of ferroptosis, inhibits ferroptosis in oligodendrocyte, finally reducing white matter injury and promoting functional recovery following spinal cord injury (SCI) in rats [48]. Additionally, investigation reveals that decreased THF lowers nucleotide biosynthesis and inhibits cell proliferation [49]. Hence, it is reasonable to believe that THF is a suitable candidate in treatment of CNS diseases as THF effectively reduces ROS cumulation that was validated in the current research.

\section{Conclusions}

In summary, the present study reveals that THF administration prominently abrogates the inhibitory effect of oxidative stress on NSC proliferation via diminishing ROS level in mouse primary NSC. And the underlying mechanism favoring of this phenomenon is that oxidative stress activates the PTEN/Akt/mTOR pathway, and PTEN serves as a negative mediator decreasing the expression of p-Akt expression and its downstream effector of p-mTOR. The present study presents a rationale for administrating THF to potentiate the rehabilitative capacity of NSC in the treatment of CNS diseases with the presence of oxidative stress.

\section{Data Availability}

The data used to support the findings of this study are available from the corresponding author upon reasonable request. 


\section{Conflicts of Interest}

The authors declare that they have no conflict of interest.

\section{Authors' Contributions}

Xuyang Zhang and Zhi Liu performed most of the experiments with assistance from Wenqin Yang, Fengchun Zhao, Chao Zhang, and Jun Zhong. Xuyang Zhang and Hui Feng analyzed the results and edited the figures. Feng Hui conducted a literature review. Tengyuan Zhou and Yongjie Zou conducted the statistical analysis. Xuyang Zhang and Wenqin Yang performed the immunoblotting and immunostaining. Xuyang Zhang wrote the preliminary draft of the manuscript. Hua Feng, Hongfei Ge, and Rong $\mathrm{Hu}$ edited the manuscript. Hongfei $\mathrm{Ge}$ and Rong $\mathrm{Hu}$ designed the experiments and revised the manuscript. All authors approved the final version of the manuscript. Xuyang Zhang and Zhi Liu contributed equally to the present work.

\section{Acknowledgments}

This work was supported by grants from the National Natural Science Foundation of China (81671228 and 82001321) and Chongqing Municipal Science Fund for Distinguished Young Scholars (cstc2019jcyjjqX0030).

\section{References}

[1] D. Ma, M. Bonaguidi, G. Ming, and H. Song, "Adult neural stem cells in the mammalian central nervous system," Cell Research, vol. 19, no. 6, pp. 672-682, 2009.

[2] J. Zhong, H. F. Ge, C. Zhang et al., "G protein-coupled estrogen receptor 1 negatively regulates the proliferation of mouse-derived neural stem/progenitor cells via extracellular signal- regulated kinase pathway," Brain Research, vol. 1714, pp. 158-165, 2019.

[3] I. Amrein, "Adult hippocampal neurogenesis in natural populations of mammals," Cold Spring Harbor Perspectives in Biology, vol. 7, no. 5, 2015.

[4] J. M. Encinas, T. V. Michurina, N. Peunova et al., "Divisioncoupled astrocytic differentiation and age-related depletion of neural stem cells in the adult hippocampus," Cell Stem Cell, vol. 8, no. 5, pp. 566-579, 2011.

[5] D. Q. Tan and T. Suda, "Reactive oxygen species and mitochondrial homeostasis as regulators of stem cell fate and function," Antioxidants \& Redox Signaling, vol. 29, no. 2, pp. 149$168,2018$.

[6] M. Bonaguidi, M. Wheeler, J. Shapiro et al., "In vivo clonal analysis reveals self-renewing and multipotent adult neural stem cell characteristics," Cell, vol. 145, no. 7, pp. 1142-1155, 2011.

[7] H. Ge, C. Zhang, Y. Yang et al., "Ambroxol upregulates glucocerebrosidase expression to promote neural stem cells differentiation into neurons through $\mathrm{Wnt} / \beta$-catenin pathway after ischemic stroke," Frontiers in Molecular Neuroscience, vol. 13, no. 263, 2021.

[8] J. Zhang, X. Jiang, C. Zhang et al., “Actin alpha 2 (ACTA2) downregulation inhibits neural stem cell migration through rho GTPase activation," Stem Cells International, vol. 2020, Article ID 4764012, 12 pages, 2020.
[9] H. Ge, A. Yu, J. Chen et al., "Poly-L-ornithine enhances migration of neural stem/progenitor cells via promoting $\alpha$-Actinin 4 binding to actin filaments," Scientific Reports, vol. 6, no. 1, p. 37681, 2016.

[10] W. Xie, J. Q. Wang, Q. C. Wang, Y. Wang, S. Yao, and T. S. Tang, "Adult neural progenitor cells from Huntington's disease mouse brain exhibit increased proliferation and migration due to enhanced calcium and ROS signals," Cell Proliferation, vol. 48, no. 5, pp. 517-531, 2015.

[11] J. E. Le Belle, N. M. Orozco, A. A. Paucar et al., "Proliferative neural stem cells have high endogenous ROS levels that regulate self-renewal and neurogenesis in a PI3K/Akt-dependant manner," Cell Stem Cell, vol. 8, no. 1, pp. 59-71, 2011.

[12] M. Khacho, A. Clark, D. S. Svoboda et al., "Mitochondrial dynamics impacts stem cell identity and fate decisions by regulating a nuclear transcriptional program," Cell Stem Cell, vol. 19, no. 2, pp. 232-247, 2016.

[13] I. Hwang, D. Tang, and J. Paik, "Oxidative stress sensing and response in neural stem cell fate," Free Radical Biology \& Medicine, vol. 169, pp. 74-83, 2021.

[14] V. S. Adusumilli, T. L. Walker, R. W. Overall et al., "ROS dynamics delineate functional states of hippocampal neural stem cells and link to their activity-dependent exit from quiescence," Cell Stem Cell, vol. 28, no. 2, pp. 300-314.e6, 2021.

[15] B. S. Xie, Y. Q. Wang, Y. Lin et al., "Inhibition of ferroptosis attenuates tissue damage and improves long-term outcomes after traumatic brain injury in mice," CNS Neuroscience \& Therapeutics, vol. 25, no. 4, pp. 465-475, 2019.

[16] S. Tang, P. Gao, H. Chen, X. Zhou, Y. Ou, and Y. He, "The role of iron, its metabolism and ferroptosis in traumatic brain injury," Frontiers in Cellular Neuroscience, vol. 14, p. 590789, 2020.

[17] S. Orellana-Urzúa, I. Rojas, L. Líbano, and R. Rodrigo, "Pathophysiology of ischemic stroke: role of oxidative stress," Current Pharmaceutical Design, vol. 26, no. 34, pp. 4246-4260, 2020.

[18] E. T. Chouchani, V. R. Pell, E. Gaude et al., "Ischaemic accumulation of succinate controls reperfusion injury through mitochondrial ROS," Nature, vol. 515, no. 7527, pp. 431-435, 2014.

[19] S. Liao, J. Wu, R. Liu et al., "A novel compound DBZ ameliorates neuroinflammation in LPS-stimulated microglia and ischemic stroke rats: role of Akt(Ser473)/GSK3 $\beta$ (Ser9)-mediated Nrf2 activation," Redox Biology, vol. 36, p. 101644, 2020.

[20] H. Quan, E. Koltai, K. Suzuki et al., "Exercise, redox system and neurodegenerative diseases," Biochimica et Biophysica Acta (BBA)-Molecular Basis of Disease, vol. 1866, no. 10, article 165778, 2020.

[21] T. Maiuri, C. E. Suart, C. L. K. Hung, K. J. Graham, C. A. Barba Bazan, and R. Truant, "DNA damage repair in Huntington's disease and other neurodegenerative diseases," Neurotherapeutics, vol. 16, no. 4, pp. 948-956, 2019.

[22] L. Liu, K. Zhang, H. Sandoval et al., "Glial lipid droplets and ROS induced by mitochondrial defects promote neurodegeneration," Cell, vol. 160, no. 1-2, pp. 177-190, 2015.

[23] G. Burgos-Barragan, N. Wit, J. Meiser et al., "Mammals divert endogenous genotoxic formaldehyde into one-carbon metabolism," Nature, vol. 548, no. 7669, pp. 549-554, 2017.

[24] B. G. Kim and H. W. Park, "Tetrahydrofolate increases suspension growth of dihydrofolate reductase-deficient Chinese 
hamster ovary DG44 cells in chemically defined media," Biotechnology Progress, vol. 32, no. 6, pp. 1539-1546, 2016.

[25] B. Widner, C. Enzinger, A. Laich, B. Wirleitner, and D. Fuchs, "Hyperhomocysteinemia, pteridines and oxidative stress," Current Drug Metabolism, vol. 3, no. 2, pp. 225-232, 2002.

[26] B. M. Rezk, G. R. Haenen, W. J. van der Vijgh, and A. Bast, "Tetrahydrofolate and 5-methyltetrahydrofolate are folates with high antioxidant activity. Identification of the antioxidant pharmacophore," FEBS Letters, vol. 555, no. 3, pp. 601-605, 2003.

[27] V. Santoro, I. Kovalenko, K. Vriens et al., "SLC25A32 sustains cancer cell proliferation by regulating flavin adenine nucleotide (FAD) metabolism," Oncotarget, vol. 11, no. 8, pp. 801$812,2020$.

[28] X. Lei and J. Jiao, "UTX affects neural stem cell proliferation and differentiation through PTEN signaling," Stem Cell Reports, vol. 10, no. 4, pp. 1193-1207, 2018.

[29] W. Liu, L. Xu, X. Wang et al., "PRDX1 activates autophagy via the PTEN-AKT signaling pathway to protect against cisplatininduced spiral ganglion neuron damage," Autophagy, vol. 17, no. 12, pp. 4159-4181, 2021.

[30] M. Laplante and D. Sabatini, "mTOR signaling in growth control and disease," Cell, vol. 149, no. 2, pp. 274-293, 2012.

[31] J. Garbern, A. Helman, R. Sereda et al., "Inhibition of mTOR signaling enhances maturation of cardiomyocytes derived from human-induced pluripotent stem cells via p53-induced quiescence," Circulation, vol. 141, no. 4, pp. 285-300, 2020.

[32] P. Devesa, F. Agasse, S. Xapelli et al., "Growth hormone pathways signaling for cell proliferation and survival in hippocampal neural precursors from postnatal mice," $B M C$ Neuroscience, vol. 15, no. 1, p. 100, 2014.

[33] Q. Liang, Z. Luo, J. Zeng et al., "Zika virus NS4A and NS4B proteins deregulate Akt-mTOR signaling in human fetal neural stem cells to inhibit neurogenesis and induce autophagy," Cell Stem Cell, vol. 19, no. 5, pp. 663-671, 2016.

[34] M. R. Bryan and A. B. Bowman, "Manganese and the insulinIGF signaling network in Huntington's disease and other neurodegenerative disorders," Neurotoxicity of Metals, vol. 18, pp. 113-142, 2017.

[35] X. Liu, P. Zhao, X. Wang et al., "Celastrol mediates autophagy and apoptosis via the ROS/JNK and Akt/mTOR signaling pathways in glioma cells," Journal of Experimental \& Clinical Cancer Research, vol. 38, no. 1, p. 184, 2019.

[36] S. Fang, X. Wan, X. Zou et al., "Arsenic trioxide induces macrophage autophagy and atheroprotection by regulating ROSdependent TFEB nuclear translocation and AKT/mTOR pathway," Cell death \& disease, vol. 12, no. 1, p. 88, 2021.

[37] Y. Yang, K. Zhang, X. Chen et al., "SVCT2 promotes neural stem/progenitor cells migration through activating CDC42 after ischemic stroke," Frontiers in Cellular Neuroscience, vol. 13, p. 429, 2019.

[38] H. Ge, L. Tan, P. Wu et al., "Poly-L-ornithine promotes preferred differentiation of neural stem/progenitor cells via ERK signalling pathway," Scientific Reports, vol. 5, no. 1, pp. 15535-15535, 2015.

[39] H. Lu, W. Chen, W. Liu et al., "Molecular hydrogen regulates PTEN-AKT-mTOR signaling via ROS to alleviate peritoneal dialysis-related peritoneal fibrosis," The FASEB Journal, vol. 34, no. 3, pp. 4134-4146, 2020.

[40] X. Chen, C. Jiang, R. Sun, D. Yang, and Q. Liu, "Circular noncoding RNA NR3C1 acts as a miR-382-5p sponge to protect
RPE functions via regulating PTEN/AKT/mTOR signaling pathway," Molecular Therapy, vol. 28, no. 3, pp. 929-945, 2020.

[41] S. Yamanaka, "Pluripotent stem cell-based cell therapy-promise and challenges," Cell Stem Cell, vol. 27, no. 4, pp. 523-531, 2020.

[42] J. Zhong, C. Lan, C. Zhang et al., "Chondroitin sulfate proteoglycan represses neural stem/progenitor cells migration via PTP $\sigma / \alpha$-actinin4 signaling pathway," Journal of Cellular Biochemistry, vol. 120, no. 7, pp. 11008-11021, 2019.

[43] M. Cui, H. Ge, H. Zeng et al., "Repetitive transcranial magnetic stimulation promotes neural stem cell proliferation and differentiation after intracerebral hemorrhage in mice," Cell Transplantation, vol. 28, no. 5, pp. 568-584, 2019.

[44] J. Yuan, H. Ge, W. Liu et al., "M2 microglia promotes neurogenesis and oligodendrogenesis from neural stem/progenitor cells via the PPAR $\gamma$ signaling pathway," Oncotarget, vol. 8, no. 12, pp. 19855-19865, 2017.

[45] D. Wang, Y. M. Chen, M. H. Ruan, A. H. Zhou, Y. Qian, and C. Chen, "Homocysteine inhibits neural stem cells survival by inducing DNA interstrand cross-links _via_ oxidative stress," Neuroscience Letters, vol. 635, pp. 24-32, 2016.

[46] S. Chakrabarti and S. S. Visweswariah, "Intramacrophage ROS primes the innate immune system via JAK/STAT and toll activation," Cell reports, vol. 33, no. 6, article 108368, 2020.

[47] B. Gutierrez, I. Gallardo, L. Ruiz et al., "Oleanolic acid ameliorates intestinal alterations associated with EAE," Journal of neuroinflammation, vol. 17, no. 1, p. 363, 2020.

[48] H. Ge, X. Xue, J. Xian et al., "Ferrostatin-1 alleviates white matter injury via decreasing ferroptosis following spinal cord injury," Molecular Neurobiology, vol. 59, no. 1, pp. 161-176, 2022.

[49] C. Moussa, N. Ross, P. Jolette, and A. J. MacFarlane, “Altered folate metabolism modifies cell proliferation and progesterone secretion in human placental choriocarcinoma JEG-3 cells," The British Journal of Nutrition, vol. 114, no. 6, pp. 844-852, 2015. 